\title{
Colloquium: Nonlinear metamaterials
}

\author{
Mikhail Lapine \\ Centre for Ultrahigh-bandwidth Devices for Optical Systems (CUDOS), \\ School of Physics, The University of Sydney, NSW 2006, Australia
}

\author{
Ilya V. Shadrivov and Yuri S. Kivshar \\ Nonlinear Physics Centre and CUDOS, Research School of Physics and Engineering, \\ Australian National University, Canberra, ACT 0200, Australia
}

(published 12 September 2014)

\begin{abstract}
This Colloquium presents an overview of the research on nonlinear electromagnetic metamaterials. The developed theoretical approaches and experimental designs are summarized, along with a systematic description of various phenomena available with nonlinear metamaterials.
\end{abstract}

DOI: $10.1103 /$ RevModPhys.86.1093

PACS numbers: 81.05.Xj, 78.67.Pt, 42.65.- $\mathrm{k}$

\section{CONTENTS}

I. Introduction

II. Modeling

A. Microscopic theory

B. Retrieval from the scattering parameters

C. Full-wave numerical calculations

D. Phenomenological approaches

III. Implementations

A. Insertion of nonlinear elements

B. Nonlinear host medium

C. Local field enhancement

D. Nonlinear transmission lines

E. Intrinsic structural nonlinearity

F. Nonlinearity based on liquid crystals

G. Quantum and superconducting metamaterials

IV. Phenomena

A. Nonlinear self-action

1. Bistability and multistability

2. Tuning and switching

3. Modulational instability

4. Nonlinear chirality and optical activity

B. Frequency conversion and parametric amplification

1. Harmonic generation

2. Parametric amplification and loss compensation 1109

3. Phase matching

4. Phase conjugation

5. Stimulated Raman scattering

6. Nonlinear subwavelength lenses

C. Surface effects

1. Nonlinear surface waves

2. Goos-Hänchen shift at a nonlinear interface

D. Nonlinear guided waves and solitons

1. Pulse propagation

2. Spatial solitons

E. Discreteness effects

F. Related topics

V. Conclusions and Perspectives

Acknowledgments

References

*ysk124@physics.anu.edu.au

\section{INTRODUCTION}

1093

1094

1094

1095

1096

1096

1097

1097

1098

1098

1100

1101

1101

1102

1103

1103

1104

1105

1106

1107

1107

1108

1110

1111

1111

1112

1112

1112

1113

1113

1113

1114

1115

1116

1116

1117

1117
The immense flow of the research in electromagnetic metamaterials, observed since the beginning of the $21 \mathrm{st}$ century, does not require much introduction these days; it has been widely popularized (Smith, 2004; Shalaev, 2007; McPhedran et al., 2011; Pendry, 2011; Zheludev, 2011; Zheludev and Kivshar, 2012) and covered in a number of books (Eleftheriades and Balmain, 2005; Caloz and Itoh, 2006; Marqués, Martín, and Sorolla, 2008; Cai and Shalaev, 2009; Capolino, 2009; Solymar and Shamonina, 2009). The subject of metamaterials, originating from a fruitful merging of theoretical and practical ideas, was a remarkable success story, and quite insightful historical accounts on that have been provided (Shamonina and Solymar, 2007; Silin, 2012). Since the term "metamaterial" first appeared in the literature in 2000 (Pendry, 2000; Smith, Padilla et al., 2000; Smith, Vier et al., 2000), numerous specific definitions have been given, expanding on the idea that metamaterials represent a structure beyond conventional materials; the way in which they are "beyond" ranges from an emphasis on having unusual properties, such as negative refraction (Pendry, 2000), to the logic of their structural hierarchy, e.g., materials made out of materials (Lakes, 1993; Lapine and Tretyakov, 2007). While the very definition of metamaterials is vaguely treated, and it was subject to some debate (Lapine and Tretyakov, 2007; Sihvola, 2007), there is no doubt that the overall impact of metamaterial research on electromagnetics, acoustics, and material science, as well as electrical and mechanical engineering, is dramatic, while the enthusiasm of numerous research teams is still far from being exhausted.

The key point in the success of metamaterials, arguably, is that they are artificial material with desirable properties, offering the freedom of using any structural components ("meta-atoms") which can be assembled with modern technology, and their deliberate arrangement to yield the envisaged material properties. This easily brings novel and unique features which are not available in natural materials, a well-known example being the negative refractive index (Mandelstam, 1945; Pafomov, 1959; Veselago, 1968; Silin 
and Sazonow, 1971), which potentially enables superresolution (Pendry, 2000), cloaking (Leonhardt and Smith, 2008), hyperbolic dispersion (Drachev, Podolskiy, and Kildishev, 2013), etc.

It was therefore quite reasonable to expect that the large realm of nonlinear optics may find a new playground in the field of electromagnetic metamaterials, so that a desired nonlinear response can be constructed depending on particular needs and enhanced as compared to conventional nonlinearity in natural materials. Similar attempts were known well before the metamaterial boom was ignited, with Kalinin and Shtykov (1990) suggesting an artificial medium for phase conjugation (an essentially nonlinear process), or Pendry et al. (1999) making a forecast for the resonant enhancement when revitalizing the use of split-ring resonators (Hardy and Whitehead, 1981), which were soon to take the role of the most famous metamaterial building block.

And indeed, the onset of explicit development occurred shortly after the metamaterial emergence, back in 2003, when the three groups independently heading in this direction introduced the concept of nonlinear metamaterials (Lapine, Gorkunov, and Ringhofer, 2003; Zharov, Shadrivov, and Kivshar, 2003; Agranovich et al., 2004). The number of publications started to escalate in 2005, and it has been growing ever since, having covered a wide range of nonlinear phenomena and suggested various practical implementations.

The key impact of metamaterials on nonlinear electrodynamics can be formulated with two aspects: (i) they allowed one to target not only electric, but also magnetic nonlinearity, as well as their combinations, and (ii) they were capable of dramatically boosting the magnitude of available nonlinearities. It is therefore not surprising that the topic has rapidly gained its popularity across a broad community in electromagnetics, promising enhanced performance and novel phenomena.

The purpose of this Colloquium is to provide an overall picture of the research on nonlinear electromagnetic metamaterials: covering both the theoretical and experimental aspects of their design and performance, introducing the developed or proposed fabrication methods, and reviewing a rich variety of exciting nonlinear phenomena in metamaterials, reported to date.

This Colloquium is organized as follows. First we offer a general perspective of the methods employed for the analysis of metamaterial nonlinearity (Sec. II), then we provide an overview of various approaches for the design of nonlinear metamaterials (Sec. III), and finally proceed toward the discussion of specific phenomena analyzed or observed in this field (Sec. IV).

We note that nonlinear metamaterials are not an isolated research topic, and there are natural links to many interesting areas, such as tunability, electromagnetically induced transparency, plasmonics, active media, etc. However, within the frame of a Colloquium we put primary emphasis on the nonlinear behavior and consider the adjacent topics only briefly, guiding the interested reader to corresponding reviews where available. With this respect, we acknowledge several earlier surveys which addressed various specific aspects in this area in more detail or pointed out certain aspects of nonlinear metamaterials within a more general context: optical nonlinearity along with a negative refractive index (Maimistov and Gabitov, 2007), microwave nonlinear phenomena with metamaterials based on transmission lines (Kozyrev and van der Weide, 2008), possible nonlinearity in photonic metamaterials (Litchinitser and Shalaev, 2008), active and tunable metamaterials (Boardman et al., 2011), wave mixing and harmonics generation (Shramkova and Schuchinsky, 2012), metamaterial nonlinearity based on liquid crystals (Zheludev and Kivshar, 2012), or superconducting metaatoms (Anlage, 2011; Zagoskin, 2011; Zheludev and Kivshar, 2012). Taking a wider perspective, the studies on nonlinear-optical metamaterials have a close relation to the research on nonlinear plasmonics, which enjoyed a recent review by Kauranen and Zayats (2012).

\section{MODELING}

Nonlinear metamaterials began on the theoretical side of research, and several years passed before practical implementations started to appear. Accordingly, theoretical description of nonlinear metamaterials was the first stage in their development. However, the approaches varied considerably and can be classified depending on the degree of approximations used and on the starting assumptions. On one pole, there are examples of metamicroscopic theory, where the analysis departs from the exact properties of the structure on the level of individual elements, often discussing a particular implementation method, and derives the corresponding effective medium parameters to describe the response of the entire metamaterial in terms of linear permittivity and/or permeability and nonlinear susceptibilities of one or several orders. On the opposite pole, theory relies on homogenized medium parameters, hypothetically assumed or retrieved from scattering, and then discusses a mixture, interplay, or perhaps further structuring of such media to obtain the overall nonlinear behavior. Most of the models reported in the field fall somewhere close to either of these two extremes, sometimes combining the advantages of each approach and using different degrees or generality. In the following sections, we review the key corresponding achievements.

\section{A. Microscopic theory}

As outlined above, the microscopic approach is built on the basis of specific properties of a particular design of metamaterial, having the theoretically firm or experimentally proven characteristics of its components as the starting point. These characteristics are already macroscopic from the point of view of conventional electromagnetism, but they play a role of microscopic features in metamaterial analysis, where a secondary averaging is performed stepping onto the next level of structural hierarchy (Lapine and Tretyakov, 2007), yielding, as we say, metamacroscopic properties.

One of the first examples of that kind of treatment was actually presented long before metamaterials were branded, by Kalinin and Shtykov (1990), who analyzed a medium of randomly oriented dipoles loaded with varactors, and derived a third-order nonlinear susceptibility of such medium in search of an all-optical phase conjugation of microwaves. Although their conclusions were pessimistic, suggesting a 
dominance of loss, the idea survived over the years to be fruitfully developed in the context of metamaterials; that was initiated with the analysis of metamaterials made of split-ring resonators loaded with diodes (Lapine, Gorkunov, and Ringhofer, 2003). In that approach, each resonator was modeled as a linear $R, L, C$ circuit loaded with a lumped nonlinear insertion with a generic nonlinear voltage-current characteristic written as $I=Y\left(U+\gamma U^{2}\right)$, which holds for weak nonlinearity $\left(\gamma \ll 1 / U_{\max }\right)$. The entire metamaterial was then modeled in the frame of quasistatic effective medium theory (Gorkunov et al., 2002), which provides explicit expressions for the linear permeability as well as nonlinear magnetic susceptibility of the second order through the parameters of metamaterial elements, the diode, and the lattice.

This kind of nonlinear metamaterial, therefore, makes use of a conventional electric nonlinearity of semiconductors, which is provided by the diode and acts as a source of metamicroscopic nonlinearity. In the metamacroscopic response of the entire structure, however, the nonlinearity manifests itself as a magnetic one. This conversion is engineered on the level of individual elements: the diode provides a nonlinear dependence of the current on the electromotive force which is induced by the magnetic field of the incident wave; then the magnetization of the metamaterial, defined by the magnetic moments of the resonators due to flowing current, acquires nonlinear dependence on the imposed magnetic field. With the majority of designs considered so far, sufficient magnetic fields for nonlinear operation should be of the order of a few $\mathrm{A} / \mathrm{m}$ or even a fraction of that, which is quite feasible in the microwave range.

Remarkably, nonlinearities provided by microwave nonlinear devices can be quite strong, featuring a much higher response than typical in optics, where the intrinsic atomic nonlinearity normally results in rather low susceptibility magnitudes. In such cases, the theoretical approach which relies on the first terms in the power-series expansion (Lapine, Gorkunov, and Ringhofer, 2003) is not applicable; however, it is still possible to model the nonlinear response, but specifically for a particular nonlinear process. For example, Lapine and Gorkunov (2004) presented an analysis of three-wave interaction in a metamaterials with nonlinearity provided by inserting a device with arbitrary nonlinear resistance and/or capacitance into each resonant ring in an array. Frequency interaction is analyzed within a nonlinear circuit, so that a relationship between the induced currents and voltages over nonlinear devices is determined from a system of coupled equations, with coefficients which can be calculated through a Fourier expansion of the device response function. The latter may be known directly from experiments and can be used in the form of either analytical functional approximations or numerically mapped functions. It is then possible to calculate the response of entire metamaterial, relating fields and magnetization at the interacting frequencies. However, it cannot be conveniently expressed in terms of susceptibility, and results in more sophisticated relations, where, e.g., the pump field is entangled within a numerically obtained term describing nonlinear modulation (Lapine and Gorkunov, 2004). Nevertheless, this is a useful modeling approach as the actual behavior of a metamaterial can be precisely described as long as the characteristics of the insertion are known, and it is applicable for handling large nonlinearity, reaching a few percent in nonlinear modulationoverwhelmingly stronger than in the classical schemes of nonlinear optics.

A similar approach was used by Zharov, Shadrivov, and Kivshar (2003) to derive the properties of another implementation of nonlinear metamaterials, assuming that an array of split-ring resonators is immersed into a host medium with Kerr-type dielectric nonlinearity. The source of nonlinearity lies again in the dielectric material properties. However, the electric field is strongly enhanced in the gaps of split rings, especially at resonance. This provides, effectively, a nonlinear capacitance $C \propto \varepsilon\left(\left|E^{2}\right|\right)$, which depends on the amplitude of the electric field in the gap according to the dielectric nonlinearity $\varepsilon=\varepsilon_{0}+\alpha\left|E^{2}\right| / E_{c}^{2}$. With this model, the microscopic response of a single element can be derived and merged with the phenomenological description of the entire medium in terms of the effective parameters. This becomes particularly interesting when the effective refractive index is negative, leading to bistability and nonlinear switching between positively and negatively refracting states (Zharov, Shadrivov, and Kivshar, 2003).

Intense development of the experimental evaluation of the nonlinear metamaterials based on the split-ring resonators (SRRs), performed in the laboratory of D. R. Smith since 2010, triggered the group to revisit the derivations for the microscopic description, getting into further details of the model assumed for an individual element (Poutrina, Huang, and Smith, 2010) and mixing analytical and numerical steps (Poutrina et al., 2011). Further work in this direction is expected to address the peculiarities of the local field arising from the mutual interaction of the elements in the lattice (Gorkunov et al., 2002; Baena et al., 2008), which would reach a wider applicability for bulk metamaterial samples.

Certain aspects of microscopic modeling are also required in the analysis of nonlinear transmission lines (Kozyrev et al., 2005) (see Sec. III.D), where highly accurate circuit models are typically used, allowing a subsequent description in terms of equivalent effective parameters. Nonlinear circuit modeling can also be applied, with certain limitations, to the analysis of bulk metamaterials, when the nearest-neighbor approximation for the mutual coupling between the elements is adopted (Colestock, Reiten, and O'Hara, 2012).

\section{B. Retrieval from the scattering parameters}

When a direct microscopic treatment is not accessible, it may still be possible to determine effective parameters without any knowledge of how the internal structure of metamaterials contributes to building up such properties. On this track, one tries to use the scattering parameters or transfer matrices, obtained experimentally or via numerical simulations, employing classical extraction methods (Nicolson and Ross, 1970; Weir, 1974), which became rather popular in the linear analysis of metamaterials (Smith et al., 2002).

It should be noted that even in the linear regime this method faces significant challenges because of the remarkable spatial dispersion typical for metamaterials (Belov et al., 2003; Silveirinha et al., 2009), ambiguity of the effective boundaries 
(Belov and Simovski, 2006; Albooyeh, Morits, and Simovski, 2011), discreteness effects (Lapine et al., 2010; Lapine, Jelinek, and Marqués, 2012), presence of higher-order modes (Andryieuski et al., 2012), and other related problems [see the comprehensive review by Simovski (2009)]. Nevertheless, retrieval procedures can provide useful insight into observable properties and can be helpful for the designs of targeting applications.

Naturally, developing a reliable and consistent retrieval procedure for nonlinear metamaterials is even more challenging due to the complex interplay between the parameters as well as to the stronger influence of spatial dispersion or bandgap effects for the harmonics. The first steps in this direction were taken by Larouche and Smith (2010) and Rose et al. (2010) in deriving the nonlinear susceptibilities from linear and nonlinear scattering data numerically obtained for nonlinear processes of a required order and considering various unit cells (Rose, Larouche, and Smith, 2011; Rose, Larouche et al., 2012). In combination with numerical methods (see Sec. II.C), retrieval procedures were used for practical design (Huang, Poutrina et al., 2011).

Retrieval approaches are particularly helpful with complex magnetoelectric structures (Smith, 2010) with entangled nonlinearities, where a microscopic analysis requires sophisticated numerical procedures, taking coupled modes into account (Rose, Larouche et al., 2012).

\section{Full-wave numerical calculations}

Apart from an analytical or semianalytical evaluation in terms of the effective parameters, a phenomenological description can rely on numerical simulations, which are based on the initial macroscopic properties of the constitutive elements (Kildishev, 2010; Kildishev and Litchinitser, 2010). For example, a Green's function suitable for nonlinear metamaterials can be derived from the generically assumed parameters (Mattiucci et al., 2005, 2010) and used for further derivations.

Numerical procedures are also important for a Fourier modal analysis, more relevant for the high-frequency range, which was used to account for either the nonlinearity with dielectric origin (Paul, Rockstuhl, and Lederer, 2010) or an intrinsic nonlinearity of the metallic fraction of metamaterials (Paul, Rockstuhl, and Lederer, 2011). In the latter work, the nonlinear response of the metallic phase was derived with the help of cold plasma equations for conduction electrons.

An alternative approach was put forward by Zeng et al. (2012). Their method is based on analyzing the eigenmodes of generic metallic structures mixed with a nonlinear dielectric substrate. The modes can be determined for arbitrarily complex structures using a finite-difference time-domain numerical simulations. At the next step, a weak nonlinear modulation is assumed, allowing corrections to the eigenmodes, arising from the nonlinear feedback, to be found with recursive calculations. This analysis appears to be quite useful to describe the nonlinear phenomena observed in metamaterials with intrinsic nonlinearity (see Sec. III.C). At the same time, its wide application is limited by the fact that it cannot be readily employed for stronger nonlinearities, common in metamaterials, and that it is rather difficult to take into account mutual interaction between individual meta-atoms, which are often much more essential in metamaterials than in conventional media.

\section{Phenomenological approaches}

The engineered nature of the nonlinear response, which allows artificial nonlinearity to be obtained while the internal source of it can be of a different nature, reflects the advantages of the metamaterial concept, providing a wide range of qualitatively new possibilities. At the same time, it facilitates a theoretical description in terms of microscopic theory, offering tools for a reliable description of the resulting media in terms of effective parameters.

A further step can then be taken by departing from such parameters as the predefined ones and analyzing various phenomena as their consequence. Once the general or typical characteristics of nonlinear metamaterials (in terms of their effective parameters) are known, it is possible to proceed to the analysis of various nonlinear phenomena on this basis.

An early general analysis of the expected outcome for nonlinear metamaterials was presented by Agranovich et al. (2004), taking advantage of describing effective media in terms of spatially dispersive permittivity $\varepsilon(\omega, \mathbf{k})$ which also includes magnetic effects as well as spatial harmonics of higher order (Agranovich and Gartstein, 2009). They used this approach to look into a nonlinear response of a medium with negative refractive index (NRI), where the properties of the latter were formally described with the corresponding linear and nonlinear material parameters, so that a microscopic analysis is bypassed. With this basis, the phenomenology of second-harmonic generation (SHG), stimulated Raman scattering, and short pulse propagation were briefly analyzed. In particular, it was pointed out that while the phase-matching conditions do not favor SHG in transmission, a strong enhancement is expected for the generation in reflection due to the counterpropagation of the fundamental and harmonic waves. The latter feature follows from a realistic assumption of a narrow-band negative refraction, so that the medium cannot possess a NRI simultaneously at frequencies $\omega$ and $2 \omega$. Efficient generation in reflection is one of the highlights of nonlinear effects in negatively refracting metamaterials, adding to their unusual physics.

Once the general form of the effective parameters in nonlinear metamaterials had been established, rapid progress was made on the route of a further phenomenological description of various phenomena, including surface waves (Shadrivov et al., 2004), band-gap properties of the onedimensional photonic crystals (Feise, Shadrivov, and Kivshar, 2004; Hegde and Winful, 2005; Mattiucci et al., 2005) or SRR structures (O'Brien et al., 2004), guided waves (Shadrivov, 2004; Boardman et al., 2005), solitons (Shadrivov and Kivshar, 2005), etc., with an established track of publications along these directions since.

With the effective parameters assumed to be predefined, a number of specific insights into general nonlinear phenomena were published, such as a general analysis of pulse propagation (Kinsler, 2010), adding nonlinearity to low permittivity metamaterials (Ciattoni, Rizza, and Palange, 2010a; Vincenti et al., 2012), analyzing cavities (Tassin et al., 2006; Martin 
and Hoyuelos, 2010), nonlocal interaction in localized structures (Gelens et al., 2007), stratified layers (K. Kim et al., 2008), or devices with supposed nonlinear metamaterial filling (Litchinitser, Gabitov, and Maimistov, 2007), and predicting the possibility of tri-refringence (de Lorenci and Pereira, 2012). In particular, increasing interest in hyperbolic media [see the special issue of Noginov et al. (2013), including the review by Drachev, Podolskiy, and Kildishev (2013)] has already stimulated theoretical analysis of nonlinear effects in hyperbolic metamaterials (Argyropoulos et al., 2013; de Ceglia et al., 2014).

We note that, in line with the phenomenological approach, an effective medium analysis can be performed without referring to a particular microscopic structure. Thus, Mackay (2005) presented an extension of the Bruggeman and Maxwell Garnett homogenization formalism, amended with the so-called strong-property-fluctuation theory [see Mackay (2005) for details and relevant references], considering a weak nonlinearity added to the dielectric properties of the structure, and reported nonlinearity enhancement for certain geometrical parameters of the composite medium. Another static consideration (Giordano and Rocchia, 2005) addressed a mixture of nonlinear dielectric particles in a linear host and revealed some shape-dependent effects, and, later, also addressed a distinction between ordered and disordered arrays of inclusions (Giordano, 2010). Static models, however, while being mathematically robust, have limited use for the main stream of nonlinear metamaterials research, as the resonant nature of most metamaterial elements requires a dynamic homogenization and may invoke spatial dispersion (Simovski, 2009).

\section{IMPLEMENTATIONS}

In this section, we present a conceptual classification of the diverse practical ways to manufacture nonlinear metamaterials. We outline the major approaches and discuss the peculiarities or specific effects associated with each implementation method. Also, individual sections are dedicated to nonlinear metamaterials made with the use of liquid crystals, and with superconducting elements, since these two topics now enjoy a remarkable attention.

\section{A. Insertion of nonlinear elements}

One of the first approaches suggested for creating nonlinear metamaterials is to use nonlinear insertions in the resonant meta-atoms of metamaterials, achieving macroscopic nonlinearity for electric (Kalinin and Shtykov, 1990) or magnetic (Lapine, Gorkunov, and Ringhofer, 2003) fields. This method is particularly convenient for metamaterials operating at longer wavelengths, where direct insertion of additional components into individual elements is technologically straightforward. In particular, this approach was developed theoretically (Lapine and Gorkunov, 2004; Sydoruk, Kalinin, and Shamonina, 2007; Maimistov and Gabitov, 2010; Yang and Shadrivov, 2010) and realized experimentally for a range of different inclusions (Shadrivov, Morrison, and Kivshar, 2006; Powell et al., 2007; Syms, Solymar, and Young, 2008; Wang et al., 2008; Powell, Shadrivov, and Kivshar, 2009b;
Huang, Poutrina, and Smith, 2010; Larouche et al., 2010; Lopez et al., 2011; Poutrina et al., 2011; Shadrivov et al., 2011). The early work presented analytical expressions for the effective nonlinear susceptibility of the metamaterials containing varactor inclusions; see Sec. II.A. This was further developed and applied to the retrieval of the nonlinear susceptibilities from experimental results (Larouche et al., 2010; Poutrina et al., 2011); see Sec. II.D.

The nonlinear response of metamaterials with nonlinear components is defined by the properties of the particular insertions; see Fig. 1(a). The first experimentally realized nonlinear split-ring resonators (Shadrivov, Morrison, and Kivshar, 2006; Wang et al., 2008) utilized varactor diodes, which have both nonlinear conductivity and nonlinear capacitance. Such properties lead to a complicated mechanism of the nonlinearity in the resonators, which is mainly caused by rectification of the induced current in the structure (Powell et al., 2007). The drawback of the nonlinear response of those structures is that the quality of the metamaterial resonance is degrading at higher powers. This was later resolved by using heterostructure barrier varactors (HBV), which have symmetric dependence of its capacitance on applied voltage, and in such structures the strength of the resonance increases with the increase of the electromagnetic wave intensity (Carbonell, Boria, and Lippens, 2008). More exotic types of inclusions are quantum dots, used along with nanoring chains (McEnery et al., 2014), or Josephson junctions, employed in the context of superconducting metamaterials (see Sec. III.G).

Bulk two-dimensional metamaterials with varactor inclusions [see Fig. 1(b)] were used by Shadrivov et al. (2008a, 2008b) and Huang, Poutrina, and Smith (2010) to demonstrate several nonlinear effects predicted for metamaterials, including harmonic generation and dynamic tunability.

Nonlinear insertions in metamaterials not only introduce the nonlinear response to the whole structure, but they can also be
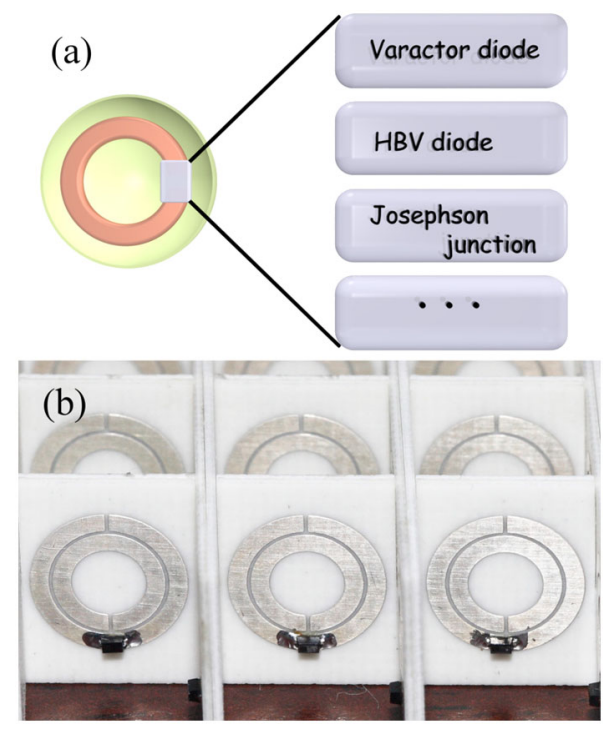

FIG. 1 (color online). (a) Schematics of a meta-atom with various possible nonlinear insertions, including a varactor diode, a heterostructure barrier varactor (HBV) diode, and a Josephson junction, and other possible diodes. (b) Metamaterial made of split-ring resonators with varactor diodes. 
used for creating meta-atoms with completely new functionality, which cannot be achieved in linear structures. In particular, it was shown that the asymmetric nonlinear meta-atom can exhibit nonreciprocal transmission of electromagnetic waves (Shadrivov et al., 2011), the so-called electromagnetic diode effect (see Sec. IV.A.4). This approach was later utilized in transmission line structures (Fan et al., 2011).

In summary, nonlinear insertions are most suitable for implementations in microwave and lower $\mathrm{THz}$ ranges and offer consistently high nonlinearities (with nonlinear modulation exceeding $10 \%$ of the linear signal) with a few watt power applied.

\section{B. Nonlinear host medium}

Making metamaterials using nonlinear insertions as described in Sec. III.A is almost impossible for optical frequencies. In optical structures it appears more feasible to use either nonlinear substrates, superstrates, or dielectric spacing layers in multilayered metal-dielectric structures as schematically shown in Fig. 2. These approaches are closely related to the idea of making nonlinear metamaterial by embedding meta-atoms into nonlinear host media (Zharov, Shadrivov, and Kivshar, 2003). Enhancement of the local electric fields (Pendry et al., 1999) near small metal components leads to the possibility of improving the nonlinear response of matter. Such an improvement would be of paramount importance, since the nonlinearities found in natural optical materials are weak and limit possible applications. We recall that both the dielectric and the magnetic properties may become nonlinear in such structures, providing an additional degree of freedom for engineering the nonlinear response of materials.

With this approach, however, it is not only possible to depart from a weak conventional nonlinearity of the host materials, achieving a stronger effect by appropriate structural arrangements, but also to have a much higher impact on the properties of host media, by affecting electronic properties through enhanced light-matter interaction. In other words, the nonlinear response of metamaterials is valuable both for employing a conventional nonlinearity on a different scale (often converting microscopic electric nonlinearity into macroscopic magnetic one) and for boosting the nonlinear

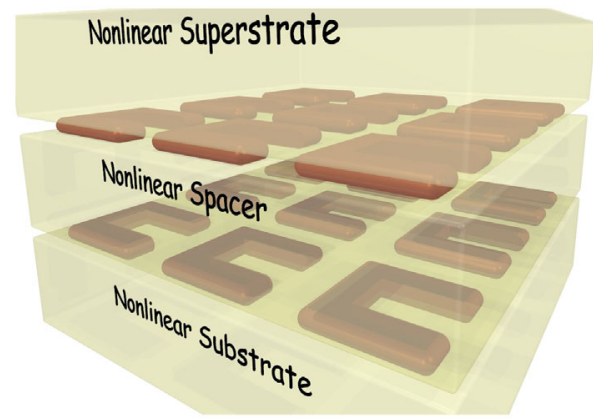

FIG. 2 (color online). Achieving nonlinear response of a metamaterial by using nonlinear substrate and/or nonlinear spacer layer and/or nonlinear superstrate. response by supplying much more intense fields to the host material.

Experimental implementations of such nonlinear metamaterials are actively being developed. The second-order nonlinear response was measured as second-harmonic generation by split-ring resonators on a GaAs substrate (Niesler et al., 2009), where specific contributions to the second-harmonic generation in the bulk substrate and from the meta-atoms were identified. A range of nonlinear phenomena was also measured for the fishnet metamaterials (E. Kim et al., 2008), where second-harmonic generation, third-harmonic generation (Reinhold et al., 2012), and four-wave mixing appear to be significantly enhanced near the resonances of the structure. Alternatively, nonsymmetric resonators can be printed on top of a nonlinear substrate (Tuz, Prosvirnin, and Kochetova, 2010).

Enhancing the response of nonlinear host media is particularly promising for $\mathrm{THz}$ metamaterials. Semiconductor substrates based on GaAs were primarily suggested to achieve tunability via photoexcitation (Padilla et al., 2006), and more recently good progress was made with this approach (Chowdhury et al., 2011; Liu et al., 2012; Fan et al., 2013), employing this technique to implement a nonlinear response with power-dependent transmission demonstrated experimentally with both doped ( $n$-type) and semi-insulating GaAs films.

More exotically, several types of superstrates were also used for achieving nonlinear response. By covering a metamaterial sample with carbon nanotubes (Nikolaenko et al., 2010), graphene (Nikolaenko et al., 2012; Rapoport et al., 2013), or liquid crystals (Minovich et al., 2012) one can achieve a dynamically tunable response.

In summary, the use of nonlinear host materials is most appropriate for $\mathrm{THz}$, infrared, and optical implementations, typically providing nonlinear response stronger than in traditional nonlinear optics, thanks to the local field enhancement and genuine combination of the components.

\section{Local field enhancement}

In the context of nonlinear metamaterials, one normally thinks of a metamaterial design, which by virtue of suitable properties and/or arrangement of the components yields an emerging nonlinear response; that is, a response which was not directly available through the use of the constituting elements alone. Albeit the most efficient and easily controllable, this is not the only possibility to use metamaterials. Instead, it is possible to seek an enhancement of a nonlinear response which originates from a conventional nonlinear material, without actually changing its nature.

Conceptually, we may think of two basic ways how this can be done. One is the use of a metamaterial structure to increase the local field in the area where nonlinearity is placed, and thus to enhance the nonlinear response. The other is to make such a structural arrangement which would improve the efficiency of a specific nonlinear process, e.g., via better phase-matching conditions.

Along the first line, Pendry et al. (1999) suggested the use of split-ring resonators a year before the concept of metamaterials was introduced, implying that a nonlinear material 
can be placed into the gaps (slits) of the resonator to benefit from the strong electric fields observed there through a resonant enhancement. Pendry et al. (1999), however, did not analyze a possible nonlinear response as such, and only a few years later Zharov, Shadrivov, and Kivshar (2003) revealed that, in fact, this design leads to an emerging nonlinearity in magnetic response rather than the enhancement of the traditional electric nonlinearity. Such an implementation, therefore, naturally borders more elaborate engineering required for nonlinear insertion (Sec. III.A) or immersion (Sec. III.B) methods.

The second approach is not really specific to metamaterials as one may easily see some analogy to, for example, phasematching approaches by use of periodically polled crystals, etc. To be more specific, we imply here that a nonlinear process enhancement is achieved by arranging individual separated structural elements, and that, ideally, mutual interaction between them is crucial for desired operation.

For example, second-harmonic generation relying on the metal surface nonlinearity can be enhanced by imposing phasematching conditions through a symmetry breaking with a specific shape of nanostructured particles in a two-dimensional array (Zheludev and Emel'yanov, 2004; Husu et al., 2008).

Substantial experimental efforts along this way were performed with T-shaped nanoparticle pairs (Canfield et al., 2007), Fig. 3 (top); L-shaped particles (Kujala et al., 2007; Husu et al., 2012) (see Fig. 12); nanorods (Wurtz et al., 2011),

Fig. 3 (bottom); and more complex patterns (Valev et al., 2011). It is clear that a particularly important role in the nonlinear processes in such structures is played by the local field effects (Husu et al., 2008), and important distinctions between surface and bulk nonlinearity contributions should be
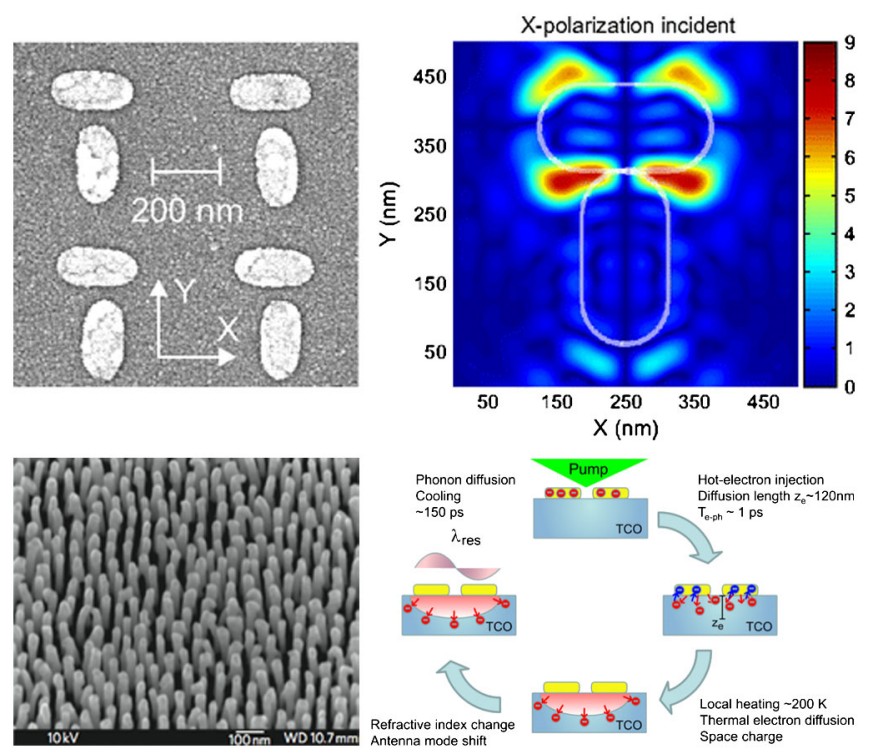

FIG. 3 (color online). Examples of metamaterials where local field enhancement is used to boost nonlinear processes. T-shaped pairs of elongated nanoparticles and polarization-specific secondharmonic enhancement. From Canfield et al., 2007. Fabricated nanorod "forests." From Wurtz et al., 2011. Emergence of the nonlinear response upon hybridization of nanoparticles with transparent conducting oxides. From Abb et al., 2012. taken into account (Wang et al., 2009). Another important aspect, arising from the challenges of nanofabrication, is related to surface defects. The influence of these can be interpreted in terms of multipolar contributions to the effective nonlinearity (Kujala et al., 2007; Zdanowicz et al., 2011). Subsequent improvement of the experimental samples eventually brought the performance to the dipole limit (Czaplicki et al., 2011), which was a key achievement for the enhancement of SHG from metal nanoparticles (Czaplicki et al., 2013), where two subsets of nanoparticles (non-centrosymmetric and centro-symmetric) were arranged in such a way that plasmon resonances of both subsets were relevant for the SHG generation.

Experimental attempts have also targeted the intrinsic nonlinearity of the metal of SRRs (Klein et al., 2006, 2007); however, these observations were complicated by spatial dispersion. The same idea was explored for the "fishnet" structures (E. Kim et al., 2008; Dani et al., 2011), which is also challenging as far as an effective description is concerned, because of the strong spatial dispersion.

Intrinsic nonlinearity of metals was also employed in nanorod structures, whether for the observation of the nonlinear magneto-optical effect in a "nanoforest" of Ni nanorods (Krutyanskiy et al., 2013) or all-optical polarization control in nanorod implementation of ENZ ( $\varepsilon$-near-zero) metamaterials (Ginzburg et al., 2013).

With metal patterns of more complex, chiral geometry, nonlinear effects may come into play for various chiro-optical effects and result, in particular, in efficient second-harmonic generation enabled by symmetry breaking (Valev, Baumberg et al., 2013).

At the same time, theoretical models were derived for a variety of structural combinations, including arrays of metallic nanoparticles with internal third-order nonlinearity (Gabitov et al., 2006), Bragg gratings made with layers of such particles (Maimistov, Gabitov, and Korotkevich, 2007), conditions for observing second-order effects in arrays of nanoparticles (Zeng et al., 2009), and nanowires (Biris and Panoiu, 2010). Alternatively, dielectric nonlinearity was studied in structural combination with a metal fraction for layers (Noskov and Zharov, 2006; Ironside and Shen, 2013), a nonlinear core with metallic shell particles (Zharov and Zharova, 2010), a combination of dielectric and metallic nonlinearity in layered structures (Scalora et al., 2010), semiconductor gratings (de Ceglia et al., 2011), binary arrays of nanoparticles with different size (Toroghi and Kik, 2012), cylindrical core-shell ENZ arrays with an "active" core and metallic nanoshell (Campione et al., 2013; de Ceglia et al., 2013), as well as sophisticated considerations like nonlinearity enhancement in material placed in the vicinity of a core-shell silica-metal nanoparticle or an array of such particles (Yannopapas, 2010). Experimental development also starts along this direction, for example, with a combination of plasmonic nanoparticles with transparent conducting oxides (Abb et al., 2012); see Fig. 3 (bottom).

In summary, the diverse range of various local field enhancement techniques employs traditional nonlinearity of conventional materials in combination with unusual properties granted by metamaterial design, which boosts nonlinearity and provides new functionality. However, special care should 
be taken with regards to the side effects of using high power with metallic structures, which can cause excessive heating and complicate the observed spectra with the effects of thermal expansion, or even damage the samples (Valev et al., 2013).

\section{Nonlinear transmission lines}

Studies of negative-index metamaterials stimulated interest to other systems supporting backward waves. In particular, socalled "left-handed" transmission lines (LH TLs) are excellent structures mimicking a backward-wave regime of metamaterials (Eleftheriades and Balmain, 2005; Caloz and Itoh, 2006). Interestingly, such TLs have low loss as compared to modern metamaterials, and as a result they are more likely to find real life applications. Such transmission lines can not only be one dimensional, but they can be made two and even three dimensional (Alitalo, Maslovski, and Tretyakov, 2006), resembling real materials.

In a left-handed transmission line, each unit cell contains series capacitance and shunt inductor; in addition, any realistic transmission line has shunt capacitance and serial inductance [see $L_{\mathrm{s}}$ and $C_{\mathrm{s}}$ in Fig. 4(a)], which are tailored along with the main components to achieve a desirable dispersion. The resulting TLs have frequency bands of negative and positive phase velocities, as shown in Fig. 4(b), and are called composite right-left-handed (CRLH) transmission lines (Eleftheriades and Balmain, 2005; Caloz and Itoh, 2006). CRLH TLs have dispersion properties very similar to those of ideal metamaterials, and therefore they offer an ideal model system for studying and verifying fascinating effects predicted for metamaterials, which are discussed in Sec. IV.

Various nonlinear effects in LH TLs were studied since 2005 (Lim, Caloz, and Itoh, 2004; Kozyrev et al., 2005; Kozyrev, Kim, and van der Weide, 2006; Kozyrev and van der
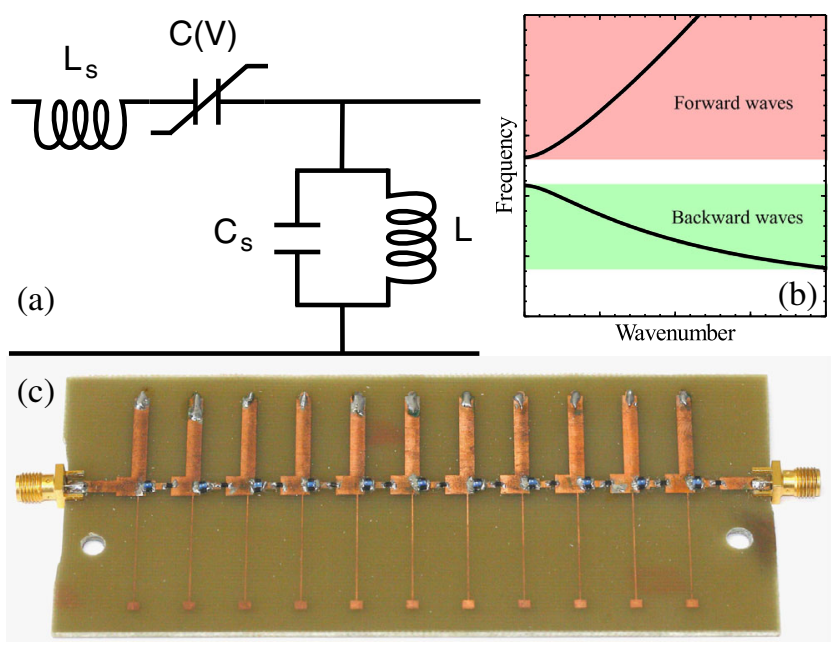

FIG. 4 (color online). (a) Schematics of a CRLH transmission line with nonlinear capacitance $C(V)$. (b) Dispersion of the CRLH TL showing the bands of frequencies where forward or backward modes are supported. (c) Nonlinear dual band CRLH TL which was used for the experiments (Somerville, Powell, and Shadrivov, 2011) with phase matching of zero phase velocity waves.
Weide, 2007, 2008, 2010; Narahara, 2007; Narahara et al., 2007; Powell, Shadrivov, and Kivshar, 2008, 2009a; Wang et al., 2010; English et al., 2011; Somerville, Powell, and Shadrivov, 2011). Usually, the nonlinearity is added to the structure by replacing capacitors with varactor diodes, which are shown as $C(V)$ in Fig. 4(a). As an additional degree of freedom, the varactors can be also biased by dc voltage to control their capacitance (Kozyrev and van der Weide, 2008).

Frequency conversion processes in the CRLH TLs can be extremely strong, due to large nonlinearities combined with strong dispersion. The crucial parameter here is phase mismatch, as discussed in Sec. IV.B.3. Normally it should be vanishing for efficient second-harmonic generation, parametric amplification, etc. In TLs, the phase mismatch can be very large, and new regimes of efficient second-harmonic generation in short transmission lines can be achieved (Kozyrev et al., 2005; Kozyrev and van der Weide, 2008). Additionally, the possibilities to engineer dispersion of waves in transmission lines allow one to obtain exotic regimes of phase matching between forward and backward waves, between two backward waves, and even between two waves with zero phase velocities (Somerville, Powell, and Shadrivov, 2011). A dual band CRLH TL supporting such unique regimes of phase matching is shown in Fig. 4(c).

Parametric amplification (see Sec. IV.B.2) is seen as one of the ways of compensating Ohmic losses in metamaterials (Popov and Shalaev, 2006a); however, it has not been observed in bulk metamaterials to date. In CRLH TLs the parametric amplification of backward waves was demonstrated in several different configurations (Kozyrev, Kim, and van der Weide, 2006; Kozyrev and van der Weide, 2008; Powell, Shadrivov, and Kivshar, 2009a). It was shown that dissipation can be completely compensated for by using parametric amplification, and, moreover, parametric generation can also be achieved.

Studies of the temporal dynamics of pulse propagation (Sec. IV.D.1) in nonlinear CRLH TLs show a rich variety of possible nonlinear phenomena. Short CRLH TLs were shown to support bright and dark envelope solitons (Kozyrev and van der Weide, 2007; Narahara, 2007; Narahara et al., 2007; Wang et al., 2010; English et al., 2011; Kozyrev, Shadrivov, and Kivshar, 2014); see Sec. IV.D.2. Remarkably, the amplitudedependent harmonic generation allows generation of short pulses with very sharp slopes.

Another potential application of nonlinear CRLH TLs is in tunable leaky wave antennas. Because of the ability to change the phase velocity of waves in tunable CRLH TLs between negative and positive values, in the leaky wave regime it becomes possible to steer the radiated beam over a large range of angles. As an example, it was demonstrated (Lim, Caloz, and Itoh, 2004) that one can not only scan the radiation angle from $50^{\circ}$ to $-49^{\circ}$, but also control beam width to a great extent. Using nonlinear transmission lines it was shown that strong electromagnetic waves can lead to the parametric generation of leaky waves, with a radiation direction which can be controlled by the pump frequency (Milford and Gibbons, 2011).

In summary, nonlinear transmission lines offer easy implementation and practical functionality, being most useful for one-dimensional waveguides or two-dimensional surface arrays. They make high nonlinearity (with the strength of 
nonlinear modulation potentially comparable with linear signal) at low power (a fraction of watt to a few watts) available for the microwave frequency range.

\section{E. Intrinsic structural nonlinearity}

An exotic way to provide nonlinearity to metamaterials relies on their internal structure rather than the properties of the materials or components. This involves a specific design that allows coupling between phenomena of different physical nature, so that the electromagnetic response is altered by mechanical or thermal effects. Such an approach essentially brings new degrees of freedom into metamaterial design and opens a wide range of possibilities, many of which are yet to be explored.

An example of such nonlinearity is found in magnetoelastic metamaterials (Lapine et al., 2012), where electromagnetic resonators are located inside an elastic medium and are therefore free to displace from their original positions (Fig. 5). This happens when the resonators are excited with an incident electromagnetic wave, which induces currents in them, so the resonators are attracted to each other by virtue of Ampère's forces and their displacement is balanced by the elastic counteraction of the host medium. The displacement, however, affects the effective permeability of the entire metamaterial through the lattice effects (Gorkunov et al., 2002). Therefore, nonlinear feedback occurs, resulting in bistability and rich nonlinear behavior (Lapine et al., 2012).

Remarkably, the nonlinear mutual coupling between the mechanical and magnetic properties in such systems originates from the specific design of the entire metamaterial, while the individual subsystems remain linear in their response. Magnetoelastic metamaterials thus provide an example of an emergent nonlinearity, where the system is built up with linear components and nonlinearity arises only through a specific interaction between them. This is quite unusual from the effective medium point of view, because the nonlinear response occurs only in the mutual interaction between the elements, but not in their individual behavior.

An interesting alternative to impose a mechanical feedback was recently put forward with a rotational degree of freedom, where a pair of split-ring resonators was connected by an elastic thread (Liu, Sun et al., 2013). This system allowed for an extremely high sensitivity in the elastic response, and remarkable rotation of the two rings with respect to each other, driven by electromagnetic forces, was experimentally observed, leading to bistable self-tuning of the resonance.
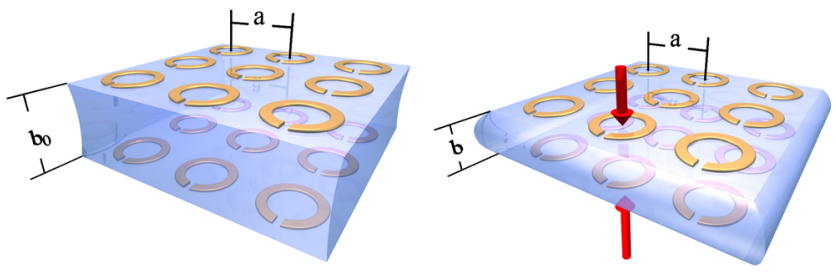

FIG. 5 (color online). Conceptual representation of a magnetoelastic metamaterial. The layers of electromagnetic resonators can be displaced due to the electromagnetic forces, induced between the element, providing a nonlinear feedback via mutual interaction in the lattice (Lapine et al., 2012).
With this design, $1 \mathrm{~W}$ power was sufficient for complete rotation; however, the response time was slow (on the scale of seconds).

The topic of metamaterial optomechanics is actively being researched, with key efforts devoted toward bringing mechanical nonlinearity to affect metamaterial response in the optical range (Zhang, MacDonald, and Zheludev, 2013), where, for example, Si nanobars were mounted onto $\mathrm{SiN}$ elastic supports to allow for structural displacement imposed by resonantly enhanced optical forces. The system possesses complex switching dynamics and bistability.

Clearly, research on magnetoelastic metamaterials is at its starting stage and a number of interesting possibilities remains to be described. In particular, accounting for dynamic interaction between electromagnetic and elastic waves in such structures should be quite fruitful for obtaining complex nonlinear behavior.

The first steps in this direction were taken on the basis of rotational nonlinearity (Liu, Sun et al., 2013), and it was demonstrated that a system of three resonators, both electromagnetically and mechanically coupled, features sophisticated nonlinear dynamics in its mechanical and electromagnetic response, with nonlinear self-oscillations and even chaotic behavior (Liu, Powell et al., 2013).

Another example where structural nonlinearity emerges is the so-called conformational nonlinearity (Lapine et al., 2011). In this case, the properties of different physical origins become connected within each individual element rather than their mutual interaction (which remains linear in this case). To demonstrate conformational nonlinearity, flexible helices have been used. They act as chiral electromagnetic resonators and at the same time as mechanical springs. When the helix is sufficiently soft, attraction between the induced currents in the windings induces a contraction of the helix which decreases the frequency of electromagnetic resonance. In addition, the helix can undergo thermal expansion due to the heating by the currents at high power, which lowers the resonance as well. These two effects provide nonlinear feedback similar to that in magnetoelastic metamaterials, and complex behavior with bistability and nonlinear chirality was observed (Slobozhanyuk et al., 2013).

Generally, various types of structural nonlinearity bring a fresh breath to the area of nonlinear metamaterials, and we should expect novel designs and approaches to appear in the near future. We should note, though, that as the mechanism of structural nonlinearity is essentially connected to the peculiarities of the design, it is quite important to use microscopic modeling to derive the material parameters.

In summary, structural nonlinearity is quite promising in terms of novel functionality and unusual effects, opening a fruitful route toward multidisciplinary designs. Possible drawbacks are relatively slow response time, and high power required for operation, which may cause excessive heating.

\section{F. Nonlinearity based on liquid crystals}

One of the specific approaches to create tunable and nonlinear metamaterials is to infiltrate a metamaterial structure with liquid crystals (Werner et al., 2007). Technically, such designs fall into the category of nonlinear host materials 
(Sec. III.B); however, peculiarities of this approach as well as its rapid development justify an individual section.

Liquid-crystal infiltration is quite attractive because of their tunable optical anisotropy and strong orientational nonlinearity. Importantly, liquid crystals offer several different approaches to realize tunability: by changing temperature, by applying external electric or magnetic field, or even all optically by employing their nonlinear-optical response (Khoo et al., 2010).

The anisotropic permittivity axes of a nematic liquid crystal follow the orientation of the director $\mathbf{n}$ associated with elongated molecules. In general, we can write

$$
\varepsilon_{i j}(\omega)=\varepsilon_{\perp}(\omega)+\varepsilon_{\mathrm{a}}(\omega) n_{i} n_{j},
$$

where $\varepsilon_{\perp}$ is the component perpendicular to the director, and $\varepsilon_{\mathrm{a}}$ is the dielectric anisotropy. External voltage applied within the metastructure gives rise to static electric fields that can reorient the liquid-crystal molecules. Minimization of the free energy of a liquid crystal in the presence of metallic inclusions gives a nonlinear equation for the orientational angle of the director that describes a change of the permittivity (Gorkunov and Osipov, 2008). For example, in a wire medium immersed into a liquid crystal, with the wires oriented along the $z$ axis, the $\varepsilon_{z z}$ component switches from $\left(\varepsilon_{\perp}+\varepsilon_{\mathrm{a}}\right)$ to $\varepsilon_{\perp}$ shifting the operational frequency $\omega_{0}$ by the relative value $\Delta \omega_{0} / \omega_{0}=$ $\varepsilon_{\mathrm{a}} / 2 \varepsilon_{\perp}$, which may reach as much as $10 \%-20 \%$.

While there are many theoretical papers which explore various concepts of metamaterial tunability and control with the help of liquid crystals (Werner et al., 2007; Kwon et al., 2008; Minovich et al., 2010), the experimental demonstrations are limited. Electrical control of the properties of microwave metamaterials infiltrated with nematic liquid crystals was experimentally shown for a periodic array of split-ring resonators (Zhao et al., 2007) demonstrating the reversible change of the transmission resonance with the maximum shift of about $210 \mathrm{MHz}$. An analogous approach was applied later to demonstrate the tunability of wire-pair (F. Zhang et al., 2010) and fishnet (Zhang et al., 2011) microwave metamaterials, where the external electric field causes reorientation of liquid-crystal molecules leading to remarkable changes in transmission. Alternatively, tunability can be achieved by applying an external magnetic field (Zhang et al., 2008, 2009).

The realization of the liquid-crystal tunability of metamaterials in the near infrared and optical regime is a much harder task; however, thermal and UV-irradiation-induced tunabilities of optical metamaterials were shown experimentally (Xiao et al., 2009; Kang et al., 2010). In the former case, the magnetic response wavelength of the metamaterial was effectively tuned through control of the ambient temperature, changing the refractive index of the liquid crystal via phase transitions. By increasing the ambient temperature from $20^{\circ} \mathrm{C}$ to $50^{\circ} \mathrm{C}$, the resonance wavelength was shown to shift from 650 to $632 \mathrm{~nm}$. As the phase transition of liquid crystals can affect the refractive index over the whole optical wavelength spectrum and even into the microwave range, it is therefore possible to tune the magnetic response of metamaterials through the whole optical range.

All-optical control of fishnet metamaterials infiltrated with E7 nematic liquid crystals was studied experimentally at the

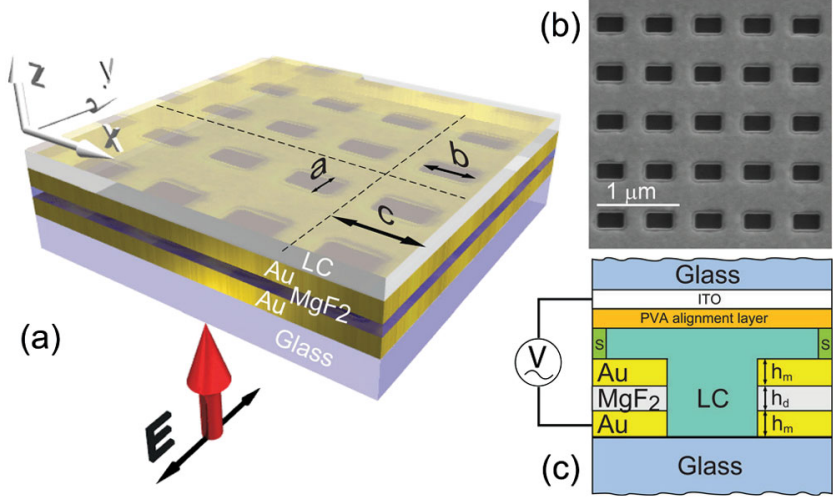

FIG. 6 (color online). (a) Schematic of the fishnet metamaterial structure infiltrated with nematic liquid crystal. (b) Scanning electron microscope image (top view) of the fabricated fishnet metamaterials. (c) Side view of the liquid-crystal cell. From Minovich et al., 2012.

telecom wavelength of $1550 \mathrm{~nm}$ (Minovich et al., 2012). In these experiments, a fishnet structure was fabricated using gold and $\mathrm{MgF}_{2}$ layers deposited on a glass substrate [Fig. 6(a)], making sure that the liquid crystal (LC) completely fills the holes of the fishnet [Figs. 6(b) and 6(c)]. They observed a power-dependent reduction in transmission by approximately $30 \%$, which was also strongly dependent on the application of a biasing electric field between the top transparent electrode and the gold film for electrical control of the LC molecular alignment [Fig. 6(c)]. This interplay between the impact of the optical fields and the biasing electric field on liquid-crystal reorientation demonstrates an interesting mechanism of electrically controlled optical nonlinearity in metamaterials, promising, for example, reconfigurable cloaking devices (Zheludev and Kivshar, 2012).

\section{G. Quantum and superconducting metamaterials}

In most of the cases studied so far, the meta-atoms of metamaterials belong to classical electromagnetics. However, recent developments in quantum mesoscopic physics, spurred by research in quantum computing and quantum information processing in solid state devices, opened a whole new field of opportunities. Fabrication of quantum bits (qubits), which maintain quantum coherence over many cycles of their internal evolution, became an experimental reality supported by quantitatively accurate theoretical models (Zagoskin, 2011), paving a road toward the so-called quantum metamaterials.

Implementation of quantum designs generally falls into the category of nonlinear insertions (Sec. III.A); however different physics of this approach as well as its active development justify an individual section.

In general, quantum metamaterials in a broad sense are artificial electromagnetic composites which (i) comprise of quantum coherent elements with desired parameters, (ii) have quantum states that can be directly controlled, and (iii) maintain global coherence for the duration of time, exceeding the traversal time of a relevant electromagnetic signal. Without the properties (ii) and (iii), the system is essentially equivalent to a 
classical metamaterial made of intrinsically quantum metaatoms. This is also valid for the so-called superconducting metamaterials (Anlage, 2011) — the most feasible candidates for the realization of quantum metamaterials.

Conventional metamaterial designs where superconductors replace metals were successfully fabricated in the microwave domain with niobium-based metamaterials, introduced by Ricci, Orloff, and Anlage (2005), and in the THz spectral domain with high- $T_{\mathrm{c}}$ superconductors (H.-T. Chen et al., 2010); however, higher frequencies destroy the superconducting phase. The low losses of $\mathrm{Nb}$ thin films at cryogenic temperatures enable metamaterials with extremely compact meta-atoms (up to $1 / 650$ of the free space wavelength), exhibiting resonances with high quality factors.

In superconducting metamaterials, resonances can be tuned by temperature (Ricci, Orloff, and Anlage, 2005; Ricci and Anlage, 2006; H.-T. Chen et al., 2010; Fedotov et al., 2010; Gu et al., 2010), external magnetic field (Ricci et al., 2007; Jin et al., 2010) or current (Savinov et al., 2012), as well as by a nonlinear response (Ricci et al., 2007; Kurter et al., 2012; Zhang et al., 2013). Remarkably, superconducting metamaterial can be naturally switched between low and high transmission by changing the power level of the incident beam, as the superconducting state can be quenched by extremely strong current densities at the corners of split-ring resonators (Kurter et al., 2012). Negative refraction in a multilayer stack of ferromagnetic and superconducting thin films was also demonstrated (Pimenov et al., 2005).

Similar to an SRR, a rf superconducting quantum interference device (SQUID) (see Fig. 7) is a resonant oscillator, exhibiting a resonant magnetic response for appropriate polarization of a time-varying magnetic field ( $\mathrm{Du}$, Chen, and Li, 2006; Lazarides and Tsironis, 2007; Zueco et al., 2013). However, the presence of a Josephson junction makes the response of such SQUID SRRs essentially nonlinear (Lazarides and Tsironis, 2007), in a way similar to the resonators with nonlinear insertions (see Sec. III.A), but with specific nonlinear dynamics. This inherent nonlinearity manifests itself at the level of macroscopic effective permeability, derived by Lazarides and Tsironis (2007) in a dipole limit (assuming a diluted array with weak interactions). Later, an analytical model of the dynamic response of the arrays of resonators with Josephson junctions was developed by

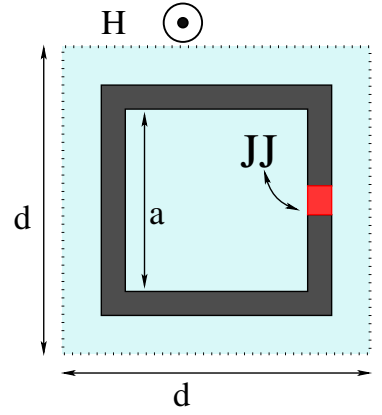

(a)

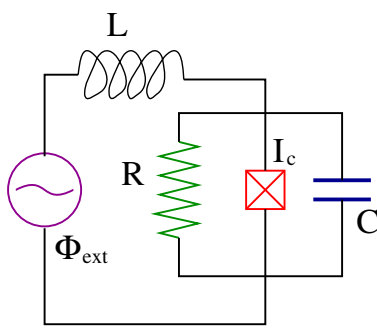

(b)
FIG. 7 (color online). Schematic of the superconducting metamaterial element based on SQUID, along with the equivalent circuit for a rf SQUID in external flux $\Phi_{\text {ext }}$. From N. Lazarides.
Maimistov and Gabitov (2010) within the nearest-neighbor approximation in planar arrays. Future work is expected to provide a more general picture for strongly interacting threedimensional arrays. In the mean time, fabricated prototypes of rf-SQUID metamaterials have shown a remarkable tunability with their frequency response controlled by a number of factors (Trepanier et al., 2013), including magnetic flux (Butz et al., 2013) or short control pulses (Jung et al., 2014).

Another example of quantum metamaterials was realized by Rakhmanov et al. (2008) with an array of superconducting qubits coupled to a transmission line, considered as point quantum scatterers. Subsequent experiments (Abdumalikov et al., 2010; Astafiev, Abdumalikov et al., 2010; Astafiev, Zagoskin et al., 2010) showed good quantitative agreement with the theory, with a single superconducting meta-atom placed in a transmission line demonstrating the effects of a quantum-optical cavity, such as resonance fluorescence and electromagnetically induced transparency. Extension of this idea into optics was suggested on a basis of quantum cavity arrays (Quach et al., 2011).

Quantum metamaterials offer a radically new paradigm for data processing and quantum information technologies, and implementations for practical devices such as electro-optical modulators (Savinov et al., 2012) are starting to appear (Zheludev and Kivshar, 2012). Superconducting metamaterials may not only provide a dramatic reduction of losses, but also allow access to the extreme sensitivity of the superconducting state to external stimuli such as heat, electric and magnetic fields, light, current, and mechanical stress. Note that the cryogenic cooling requirement for superconductors is no longer a serious technological limitation as compact cryogenic devices are now widely deployed in telecommunications and sensing equipment.

\section{PHENOMENA}

In this section, we consider various specific features and phenomena, predicted or observed in nonlinear metamaterials. While we attempt to provide a classification in accordance to the below sections, we admit that many effects can be simultaneously analyzed and are in close relation to each other, so a unique classification is not always possible. Some of the topics, which emerged in the context of nonlinear metamaterials but have far-reaching implications (for example, the vast research area of metamaterials tunability), may not be exhaustively treated in the frame of this review, and we discuss only the aspects relevant to nonlinear behavior.

As an opening remark, we recall that the most prominent features of nonlinear metamaterials are the availability of exceptionally strong nonlinearities (sometimes exceeding $10 \%$ nonlinear modulation), the translation of microscopic electric nonlinearity into a macroscopic magnetic one, and the possibility of efficient dispersion engineering. We now discuss how the advantages of metamaterials manifest themselves in nonlinear phenomena.

\section{A. Nonlinear self-action}

Among various nonlinear effects, the most straightforward one is, generally speaking, associated with the "first 
harmonic," in other words, representing a nonlinear self-action where, by virtue of nonlinear properties, the linear parameters of metamaterials are affected. This may result in switching of a metamaterial between different states, leading to bistable and multistable behavior, and can also provide continuous tuning of the linear properties with power. Next we review the work done in these directions; note that these effects are normally closely connected, so separating them into subtopics does not imply a strict distinction.

\section{Bistability and multistability}

The conceptual origin of bistability in resonant structures relies on the fact that the frequency of the resonance may shift with the change of applied power, and if the dependence is sufficiently strong, the resonance effectively "bends," yielding nonstable configurations. This results in jumps between different branches of the multivalued response function, which can be observed with varying amplitude or frequency, so that for a given input intensity, two output intensity levels can be observed (Winful, Marburger, and Garmire, 1979; Soljačić et al., 2002).

Bistable behavior is therefore quite natural for strong nonlinearity of metamaterials and was predicted at the earliest stage of research (Zharov, Shadrivov, and Kivshar, 2003), when a bistable switching between the states with positive and negative permeability was found to occur with the fields required to be of the order of tens of $\mathrm{A} / \mathrm{m}$. Further results in this direction were later obtained assuming dielectrics with saturable nonlinearity (Maluckov et al., 2008). Similar expectations on the resonance frequency bistability were also reported for an array of the SRRs scaled to optical dimensions (O'Brien et al., 2004); however, the required fields in the $\mathrm{kA} / \mathrm{m}$ range as well as inapplicability of the simple circuit models in that frequency range put the latter analysis out of practical reach.

With doubly nonlinear metamaterials, where the nonlinearity is simultaneously provided to the electric and magnetic effective response (Yang and Shadrivov, 2010), bistable behavior can be observed for both the permittivity and permeability dependence on the input power.

Using phenomenological analysis (Sec. II.D), remarkable bistability was predicted for a wide variety of (quasi)periodic band-gap structures of the alternating layers of positively or negatively refracting media with a nonlinear defect (Feise, Shadrivov, and Kivshar, 2005; Wei et al., 2008), or a mixture of nonlinear "positive" and linear "negative" layers (Hegde and Winful, 2005; Cavalcanti et al., 2014), which could be used in the infrared or optical range by combining thin metallic sheets with nonlinear dielectric spacers, resulting in diverse patterns of optical bistability (Noskov and Zharov, 2006). Further variations of such analysis include considering a single pair of a nonlinear dielectric and a linear negative-refracting layer in the dielectric environment (Litchinitser et al., 2007), cavities filled with a mixture of nonlinear positive- or negative-index materials (Kockaert et al., 2006), nonlinear properties of $\epsilon$-near-zero metamaterials (de Ceglia et al., 2013), or even devices, such as optical couplers with nonlinear positive and negative-index channels (Litchinitser, Gabitov, and Maimistov, 2007; Coelho, Jr. et al., 2013).
A convenient platform for studying bistable phenomena is offered with a TL implementation of metamaterials, where a diode insertion into each unit cell leads to multiple bistability ranges with an increase of input power, and may result in a chaotic behavior (Powell, Shadrivov, and Kivshar, 2008). TL implementations are quite fruitful in terms of both the experimental realization and the ease of precise analysis (Sec. III.D).

Later on, microscopic analysis, within a nearest-neighbor approximation for the mutual coupling, was performed to derive scattering properties and nonlinear susceptibility for arrays of SRRs loaded with Josephson junctions (Maimistov and Gabitov, 2010), reporting interesting self-induced magnetization dynamics and bistable magnetization dependence on the incident field. The arising multistable behavior was recently observed in the experiments on the transmission lines loaded with Josephson junctions (Jung et al., 2014).

Planar arrays of asymmetric SRRs of sub- $\mu \mathrm{m}$ dimensions were analyzed with the method of moments by Tuz, Prosvirnin, and Kochetova (2010), and they reported a strong optical bistability originating from a nonlinear response provided by substrate nonlinearity. Experimentally, Wang et al. (2008) explicitly reported a bistable transmission with forward and reverse frequency sweep and also considered a coupling between two varactor-loaded SRRs. Further
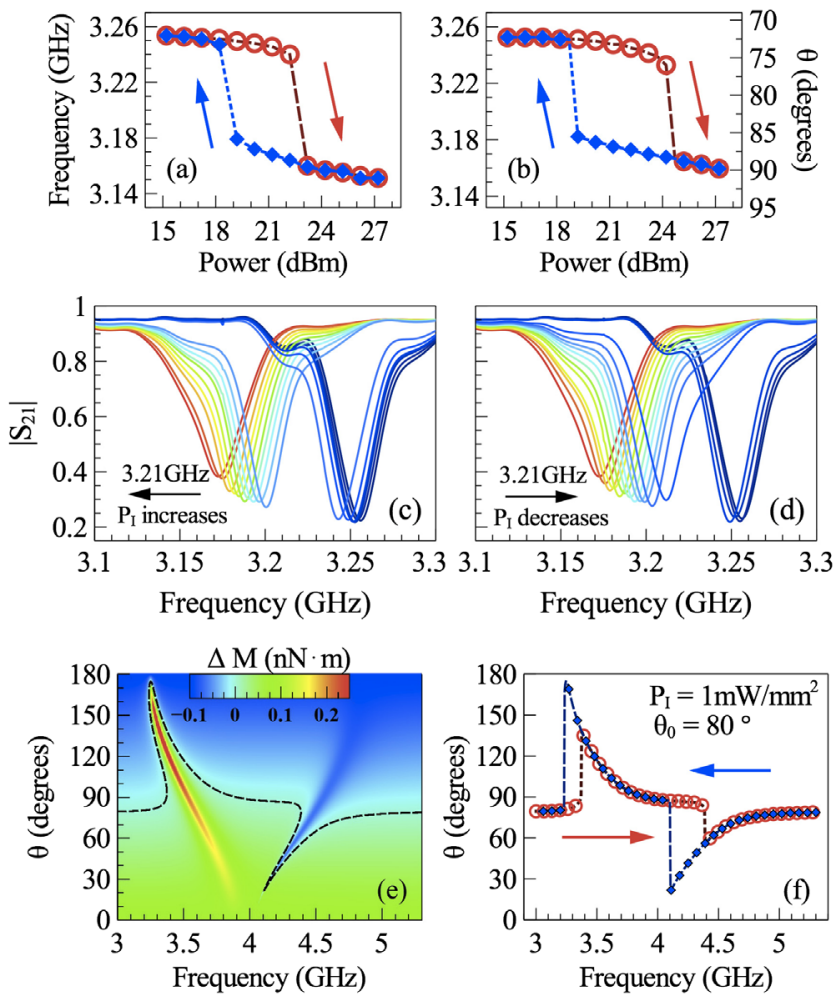

FIG. 8 (color online). Bistability in metamaterials with intrinsic rotation: (a) Experimental observation and (b) numerical confirmation of power-dependent bistability of the resonance, experimental transmission spectra of the bistable behavior for (c) increasing and (d) decreasing power, (e) calculated spectral map of the intrinsic torque in rotational meta-atoms at various twist angles, and (f) angular bistability of the twist angle depending on frequency (Liu, Sun et al., 2013). 
theoretical research on nonlinear effects in dimer SRRs with different orientational coupling and imposed nonlinearity (Lazarides et al., 2010) predicted a complex pattern of multistability and chaotic response; however, those preliminary results were obtained with a simplified qualitative model and revisiting that analysis with a more rigorous treatment (Powell et al., 2011) is desirable.

In optics, a bistable refractive index was numerically predicted (Chen, Farhat, and Alu, 2011) for an array of square silver patches with diagonal slits filled by nonlinear media.

So far, bistability phenomena were mostly treated from a theoretical or numerical perspective. Indeed, this area is particularly challenging for experimental research as the required power is typically high so stability control is necessary to avoid chaotic behavior, self-generation, and various side effects. Nevertheless, recent advances with magnetoelastic metamaterials (Sec. III.E) permitted direct experimental observation (Liu, Sun et al., 2013) of rotational bistability in a metamaterial with an internal mechanical degree of freedom, where the SRRs, connected through elastic feedback, were allowed to twist with respect to each other (Fig. 8).

Further analysis (Liu, Powell et al., 2013) predicts that a system of three resonators, which are both electromagnetically and mechanically coupled, will offer much more sophisticated nonlinear dynamics in the mechanical and electromagnetic response, leading to nonlinear self-oscillations and chaotic behavior.

\section{Tuning and switching}

Contrary to the research on multistability, which is primarily driven by academic interest, tunability is a much more practical outcome of nonlinear self-action. With tunable nonlinear metamaterials, one aims to control and adjust the linear properties with power, and this is a clear target for applications. At the same time, tuning the nonlinear properties themselves is also an interesting point to consider.

Tunability of electronic structures is an old topic in electrical engineering; it is relatively straightforward to realize with the help of additional biasing circuits which control the properties of nonlinear elements. Thus, inserting an externally biased varactor into an SRR allows its resonance frequency to be shifted with applied voltage (Reynet and Acher, 2004; Shadrivov, Morrison, and Kivshar, 2006), while incorporating varactors into a leaky wave antenna, designed as a transmission-line metamaterial, results in its radiation angle and beam width being tunable with electronics (Lim, Caloz, and Itoh, 2004).

On the other hand, it is quite natural to aim at so-called "all-optical" solutions, where self-tunability can be achieved with incident electromagnetic waves, making a direct use of nonlinearity. In the context of microwave metamaterials, such an approach was theoretically analyzed by Gorkunov and Lapine (2004), assuming various nonlinear insertions into SRRs and calculating the change of the effective permeability of metamaterial resulting from the dynamic self-biasing of the insertions. Having a nonlinear resistance results in a drop in transmission with the increasing power, while using a nonlinear capacitance shifts the resonance frequency, permitting a
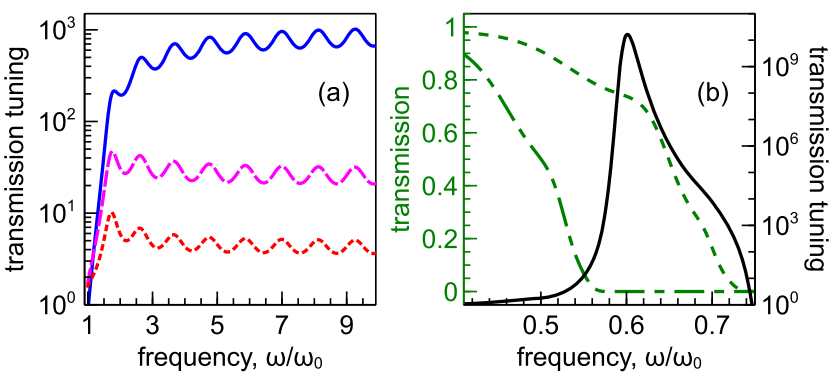

FIG. 9 (color online). Theoretical prediction (Gorkunov and Lapine, 2004) for tunability in metamaterials based on SRRs loaded with (a) backward diodes or (b) varactors. Relative transmission change for different power levels [(a) dotted, dashed, solid; (b) solid, right axis], as well as the corresponding transmission [(b) dashed and dash-dotted, left axis]. Frequency is normalized to the resonance at lower power $\omega_{0}$.

metamaterial slab to be tuned between transmission, absorption, and reflection (Fig. 9).

This approach was first implemented experimentally with a single metamaterial element by Shadrivov, Morrison, and Kivshar (2006) and further by Powell et al. (2007), who also found an interesting side effect of the zero harmonic action: it turned out that the SRR capacitors accumulate electrical charge which provides an additional biasing, persistent over repeated experiments, and complicating observations of the resonance shift significantly. To avoid this uncontrollable effect, it is necessary to shunt the varactor with a parallel inductive coil, which acts as low-pass filter to let the dc current passing over the varactor to prevent it from self-biasing with accumulated charges. Similar measurements on a varactorloaded SRR tunability and on the pair of such elements were later reported by Wang et al. (2008), who also observed a bistable transmission.

Next Shadrivov et al. (2008a) extended the experiments by fabricating a metamaterial sample with varactor-loaded SRRs and metallic rods and measuring tunable transmission characteristics (and harmonics generation, see Sec. IV.B.1). More recently, Huang, Poutrina, and Smith (2010) reproduced such measurements on a metamaterial sample and also loaded one SRR with two counterdirected varactors, which resulted in a reversed sign of the frequency shift with power.

Analogous transmission tunability was also achieved by inserting varactors into compact electric resonators (Powell, Shadrivov, and Kivshar, 2009b), where the main mode has the magnetic response suppressed and a nonlinearity is present in the electric dipole moment, while at a higher frequency the magnetic mode is approximately linear.

Efforts are ongoing to extend nonlinear tunability toward the optical range, with examples employing split metal particles filled with nonlinear layers (Chen, Farhat, and Alu, 2011), or arranging quantum dots next to nanoparticles in a resonating ring chain (McEnery et al., 2014).

For the THz range, Rahm, Li, and Padilla (2013) recently reviewed the available modulation techniques.

Nonlinear tuning was also addressed with hyperbolic metamaterials, with the intensity dependence of the transmission analyzed for third-order nonlinearity of the dielectric layers (Argyropoulos et al., 2013). 
An interesting approach to tunability is offered by using photosensitive semiconductors or photodiodes as nonlinear insertions. The initial implementations reported substantial transmission modulation in the $\mathrm{THz}$ range (Padilla et al., 2006) or at microwave frequencies (Degiron, Mock, and Smith, 2007), both being mostly concerned with the tunability in the sense of the gradual elimination of the resonance by providing photoexcited conductivity across the resonator slits. Further work (Boulais et al., 2008) claimed, in contrast, that an extremely wide-band resonance tuning is possible through varying the width of the carrier-depleted region of photoconductive GaAs, although the giant effect they reported was not yet consistently explained. Later on, experimental observation of power-dependent transmission was reported for $\mathrm{THz}$ metamaterials assembled with doped (n-type) and semiinsulating GaAs films (Fan et al., 2013). It was observed that the doped films feature a nontrivial trend in carriers dynamics, reversing at about $160 \mathrm{kV} / \mathrm{cm}$ fields, so that the initial decrease in the transmission at moderate power is followed by an increase at higher power. For the undoped substrates, to the contrary, the trend was uniform (Fan et al., 2013).

In the microwave range, a system with gradient optical control was designed by using SRRs loaded with photodiodes (Kapitanova et al., 2011), which allowed for optically tunable steering, focusing, and defocusing of the microwaves (Shadrivov et al., 2012). This technique also resulted in competing nonlinearities (Kapitanova et al., 2012), with a curious interplay between the nonlinearity of the varactors with that of the photodiodes.

Furthermore, by including both light-emitting and photosensitive diodes within a specially designed SRR pair, it was possible to establish a nonlinear-optical bridge between the microwave resonators, so that the signal power in one of the rings affects the resonance of the other, in spite of the absence of direct magnetic coupling between the two (Slobozhanyuk et al., 2014).

Where the dissipation in metamaterials becomes affected instead of the resonance frequency [for example, with the use of resistive nonlinearity (Gorkunov and Lapine, 2004)] nonlinear absorbers can be designed, allowing power filtering to prevent damage to sensitive receivers (Katko et al., 2011; Sievenpiper, 2011).

On the side of practical applications, a slab of nonlinear metamaterial was suggested to improve the signal-to-noise ratio for magnetic resonance imaging applications (Lopez et al., 2011), by virtue of power-dependent switching of the effective permeability between the values close to 0 and close to -1 .

Also, nonlinear self-tuning may provide a wide frequency range with negative material parameters (Lapine, Shadrivov, and Kivshar, 2012), for which a remarkable nonlinear shift of the resonance is required.

Apart from the use of nonlinearity to tune metamaterial properties with power, other tuning approaches can be employed to modify the nonlinear response, for example, by applying structural tuning (Powell et al., 2010) to control the nonlinear properties of symmetric and antisymmetric modes in the varactor-loaded coupled resonators (Hannam et al., 2012).

\section{Modulational instability}

Discussing nonlinear metamaterials, we are mostly talking about steady-state phenomena. However, it is well known that nonlinearity often leads to nontrivial time-dependent processes. It is natural to expect that nonlinearity in metamaterials would give rise to a number of instability scenarios.

The question of stability naturally emerges (Lazarides and Tsironis, 2005; Scalora et al., 2005; Maluckov et al., 2008) in the context of pulse propagation (Sec. IV.D.1) and was initially addressed by Kourakis and Shukla (2005) for a generic pulse propagation scheme, followed with a more specific analysis of modulational instability performed by Kockaert et al. (2006) for oscillations in a cavity, and by Wen et al. (2006) for short pulses. Such analysis is typically assuming a negative refractive index and a Kerr nonlinearity and relies on a generic phenomenological description.

The latter group has published a number of follow-up works, concentrating on various specific aspects, such as dispersion effects (Xiang et al., 2007), copropagating pulses (Dai et al., 2009), higher-order dispersion (Zhang, Wen, Xiang, and Luo, 2010), saturable nonlinearity (Xiang et al., 2011), as well as grasping various nonlinearity implementations or suggestions for applications published in contemporary literature [see the example by Xiang et al. (2010), where a nonlinear directional coupler of Litchinitser, Gabitov, and Maimistov (2007) is assessed against possible modulational instability; see Fig. 10]. A few alternative treatments were presented by other groups (Joseph, Porsezian, and Tchofo Dinda, 2010; Sarma and Saha, 2011), also relying on a generic description with a negative index and Kerr nonlinearity implied.

The phenomenological nature and somewhat mathematical scope of the typical contributions in this area limit their practical outreach. In the future, it would be useful to consider such phenomena within the frame of microscopic analysis that would take the actual structure of metamaterials into account. This, however, is a challenging task as severe complications arising from various types of disorder (Gorkunov et al., 2006;
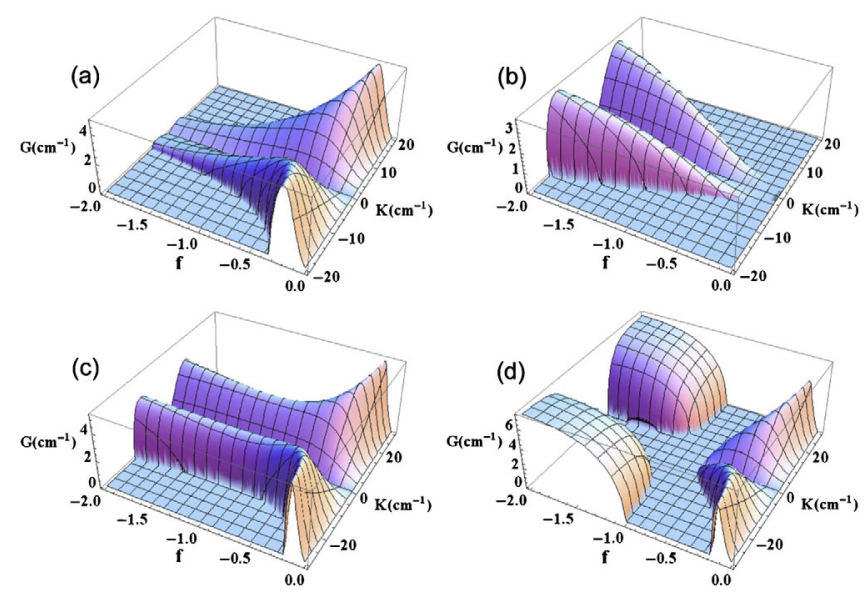

FIG. 10 (color online). Modulational instability scenarios calculated for a nonlinear directional coupler with a negative refractive index channel. The spectral gain $G$ shown for various forward to backward power flow ratios $f$ and different wave vectors $K$. From Xiang et al., 2010. 
Rico-García, López-Alonso, and Aradian, 2012) and noise (Syms and Solymar, 2011; Syms, Sydoruk, and Solymar, 2011) will also influence the temporal dynamics of nonlinear processes.

\section{Nonlinear chirality and optical activity}

A power-dependent chiral response represents an interesting and as yet exotic area for the application of nonlinearity to control the linear properties of metamaterials. Optical activity depends on light intensity and therefore nonlinearity-induced polarization rotation can be observed in nonlinear crystals such as lithium niobate. However, such effects are very weak in natural crystals but can be enhanced in structured media such as metamaterials.

Recently, a strongly nonreciprocal nonlinear element, a chiral electromagnetic diode, was demonstrated (Shadrivov et al., 2011), which is a direct analog of an electronic diode. The effect exploited in this electromagnetic diode is an intensity-dependent polarization change in an artificial chiral meta-atom. This microwave effect exceeds a similar optical effect previously observed in natural crystals by more than 12 orders of magnitude and a direction-dependent transmission that differs by a factor of 65 . The rotatory power of the sample is comparable to that obtained earlier in an artificial structure (Rogacheva et al., 2006), and it is 2 orders of magnitude stronger than the rotatory power of cholesteric liquid crystals, sculptured thin films (Hodgkinson et al., 2000), and chiral metamaterials for optical wavelengths (Kuwata-Gonokami et al., 2005).

Further studies have experimentally demonstrated strong cross-phase modulation and four-wave mixing in a chiral metamaterial, highlighting the interplay of nonlinearity and circular dichroism (Rose et al., 2013). It was shown that the magnitude of the nonlinear parametric interaction follows certain selection rules regarding the circular polarization of the various interacting waves. Using a coupled mode analysis and finite element simulations, these selection rules are linked to the metamaterial's internal symmetries as well as its circular dichroism in the linear regime. A convenient planar design of the canonical spirals was presented (see Fig. 11), which offers a great potential for engineering of the electric, magnetic, and chiral properties of the nonlinear metamaterials.

A similar effect was reported for plasmonic chiral metamaterials [see Valev, Baumberg et al. (2013)] exhibiting a large nonlinear-optical activity in the optical part of the spectrum (Ren et al., 2012), claimed to be $30 \times 10^{6}$ times stronger than that in lithium iodate crystals (Akhmanov et al., 1979). As a result, nonlinear rotation on the order of degrees has been observed at a laser power of only a few milliwatts (Ren et al., 2012).

Another plasmonic approach (Krutyanskiy et al., 2013) leading to nonlinear magneto-optical activity employed the natural magnetic response of Ni nanorods, which were structured into a nanoforest to bring plasmonic resonances into play.

Notably, in plasmonics chiral structures are also used to provide symmetry breaking necessary for second-harmonic generation (Sec. IV.B.1); reciprocally, SHG is employed to assess the chirality (Huttunen et al., 2011).

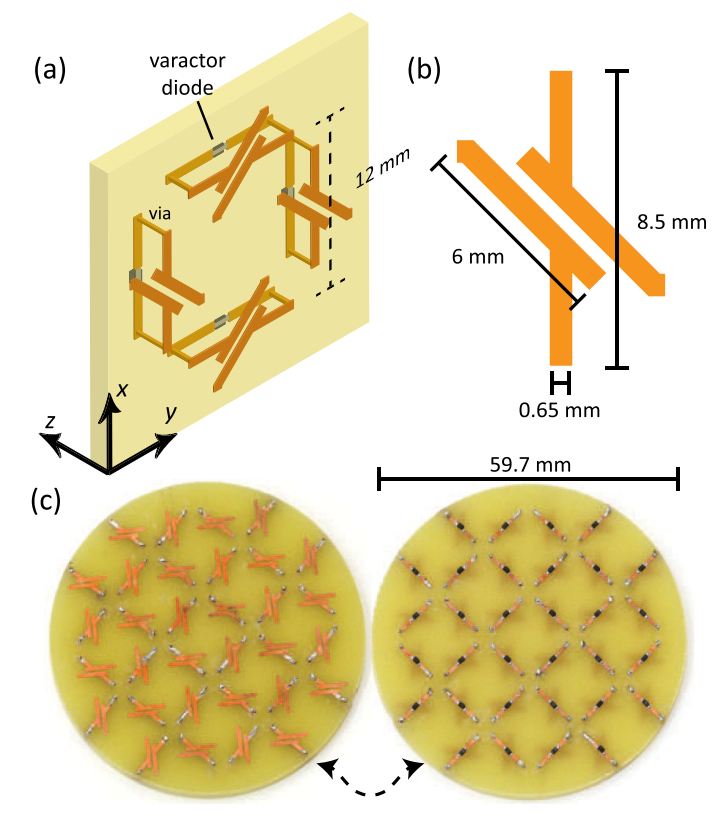

FIG. 11 (color online). (a) Metamaterial unit cells each consisting of two nonlinear particles; (b) details of one side of the nonlinear particle, with (c) photographs of the two sides of the fabricated sample. From Rose et al., 2013.

A recent microwave design by Shadrivov (2012) offered a purely nonlinear-optical activity, where a racemic mixture of canonical spirals is used, where all the spirals with a given handedness are loaded with a nonlinear insertion. At low power, the two spirals have the same impedance and chirality is compensated; however, with the increasing power the two subsets are not equivalent anymore and a differential optical activity emerges.

To dramatically enhance nonlinear bianisotropy, generally observed with most of the structural elements, Rose, Huang, and Smith (2012) suggested using double-split rings with the two varactors which can have either the same or the opposite direction, providing for the power-dependent splitting between the modes.

A novel approach to obtain power-dependent chirality relies on structural nonlinearity (see Sec. III.E). This can be achieved with flexible helices for metamaterials (Lapine et al., 2011; Slobozhanyuk et al., 2013), whereby multiturn helices (Slobozhanyuk et al., 2013) appear to be particularly useful, as they show both a stronger chirality and a stronger nonlinear response. The chirality of each helix directly depends on its pitch, so the mechanical contraction commencing with increasing power decreases the chirality coefficient. This may lead to interesting behavior related to the wave propagation inside the bulk samples of such metamaterials, leading to nonlinear polarization rotation and formation of dynamic domains with different chirality inside the initially homogeneous metamaterial. Such complex phenomena remain to be explored in the future.

\section{B. Frequency conversion and parametric amplification}

Two of the most widely used nonlinear effects are harmonic generation and parametric amplification. These processes 
involve energy exchange between waves of different frequencies, and this becomes possible due to nonlinear properties of the media supporting the interacting waves (Boyd, 2003). In metamaterials, due to nontrivial linear and nonlinear properties, these processes are significantly different as compared to natural dielectrics, and we overview them next.

\section{Harmonic generation}

Second-harmonic generation, also known as frequency doubling, is the generation of an electromagnetic wave with a frequency twice that of the incident wave. It is a particular case of a three-wave mixing, the amplitudes of the interacting waves are governed by (Boyd, 2003)

$$
\begin{aligned}
& \frac{d h_{1}}{d z}=i d_{1} h_{2} h_{1}^{*} \exp (-i \Delta k z), \\
& \frac{d h_{2}}{d z}=i d_{2} h_{1}^{2} \exp (i \Delta k z),
\end{aligned}
$$

where $h_{1,2}$ are the amplitudes of fundamental frequency (FF) wave and second harmonic (SH), respectively, $\Delta k=2 k_{1}-k_{2}$ is the phase mismatch, $k_{1,2}$ are the wave numbers of the FF and $\mathrm{SH}$ waves, $d_{1,2}$ are coefficients depending on the nonlinear susceptibility of the material, and $z$ is the propagation direction. The energy conversion crucially depends on $\Delta k$, and the best results can be achieved in the perfect phasematching regime, when $\Delta k=0$. This condition is particularly hard to find in natural materials, and we discuss it for the case of metamaterials separately in Sec. IV.B.3.

In metamaterials, second-harmonic generation was theoretically considered in a number of works (Agranovich et al., 2004; Lapine and Gorkunov, 2004; Mattiucci et al., 2005; D'Aguanno et al., 2006; Gorkunov, Shadrivov, and Kivshar, 2006; Popov, Slabko, and Shalaev, 2006; Scalora et al., 2006, 2010; Shadrivov, Zharov, and Kivshar, 2006; Roppo et al., 2007, 2008, 2010; de Ceglia et al., 2007, 2011; Centeno and Ciraci, 2008; Chowdhury and Tataronis, 2008; Zeng et al., 2009; Biris and Panoiu, 2010; Popov, 2010; Paul, Rockstuhl, and Lederer, 2011; Popov and Myslivets, 2011). Already in the early work of Agranovich et al. (2004) it was predicted that the second-order nonlinear effects in dispersive negativeindex media would be very unusual, with possible strong interaction of forward and backward waves.

Experimental verifications of the predicted nonlinear interactions are still rather scarce, with most results demonstrated for the microwave metamaterials (Shadrivov et al., 2008b; Huang, Rose et al., 2011; Nakanishi, Tamayama, and Kitano, 2012) and for the left-handed transmission lines (Kozyrev et al., 2005; Kozyrev and van der Weide, 2010; Somerville, Powell, and Shadrivov, 2011). Quantitative characterization of the quadratic nonlinear response of the microwave metamaterials has been presented by Larouche et al. (2010). Optical experiments are technologically much more complex, and most of the works study only single functional layer structures (E. Kim et al., 2008; Niesler et al., 2009; Wang et al., 2009; Kauranen and Zayats, 2012), where phasemismatch effects are negligible.

Because of the narrow frequency band, where the index of refraction is negative, the interaction of the fundamental frequency and second harmonics takes place for the waves with energy propagating in opposite directions, for which exact phase matching can be achieved (Popov, Slabko, and Shalaev, 2006; Shadrivov, Zharov, and Kivshar, 2006). Remarkably, such interaction of a backward FF wave and a forward SH wave leads to second-harmonic generation in the reflected wave [see Fig. 12(a)], and for the case of the semiinfinite metamaterial, it will form a second-harmonic reflecting mirror (Popov, Slabko, and Shalaev, 2006; Shadrivov, Zharov, and Kivshar, 2006; Popov, 2010; Popov and Myslivets, 2011). Accordingly, energy conservation in the lossless case is written in the somewhat unusual form (Popov, Slabko, and Shalaev, 2006; Popov, 2010),

$$
\frac{d}{d z}\left(\left|h_{1}\right|^{2}-2\left|h_{2}\right|^{2}\right)=0
$$

Indeed, for conventional dielectric media, a similar energy conservation relation, known in optics as the Manley-Rowe relation, is written with a plus sign, having the clear meaning that the sum of the energies carried by the FF and $\mathrm{SH}$ waves is conserved. In negative-refracting metamaterials, however, because of the counterpropagation of the energy in interacting waves, the difference of the energies is conserved. This means that two photons at the FF convert into an oppositely propagating SH photon, and both waves have their amplitudes decaying in the same direction, as shown in Fig. 12(a). Similar conditions of phase matching were predicted for layered structures and photonic crystals long ago (Bloembergen and Sievers, 1970) and observed experimentally (van der Ziel and Ilegems, 1976), but for the metamaterials we expect much more flexibility for designing such second-harmonic generation.

An unusual property of the SHG process is predicted for the case of a finite thickness nonlinear negative-index slab which is

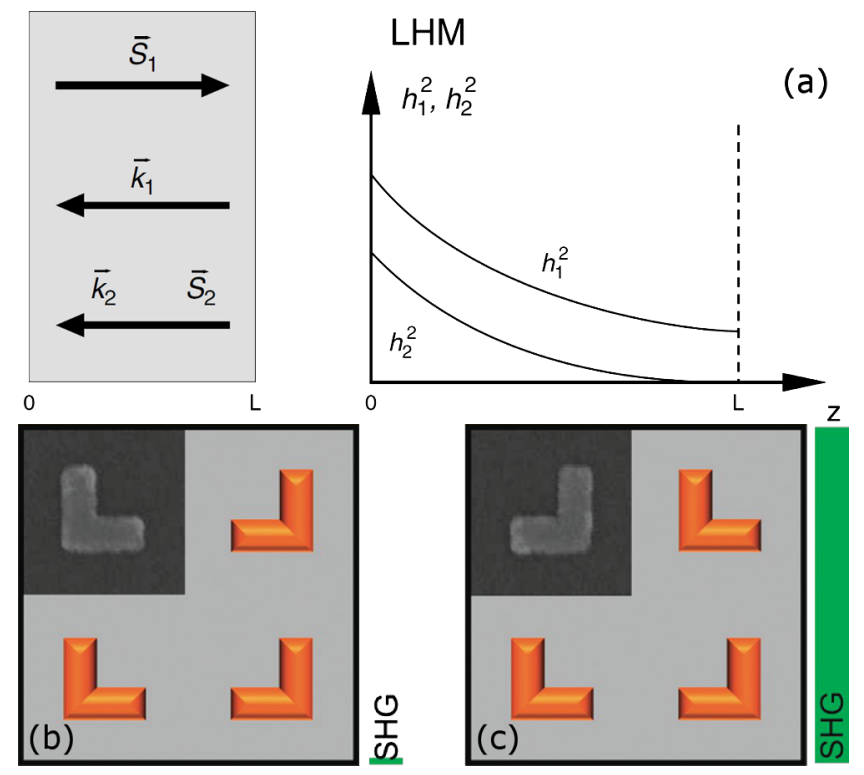

FIG. 12 (color online). (a) The direction of energy flows and wave vectors of FF and $\mathrm{SH}$ waves in a slab of metamaterial and amplitudes of the corresponding waves $h_{1,2}$. From Popov, Slabko, and Shalaev, 2006. (b), (c) Plasmonic nanoparticles arranged in a periodic lattice with different symmetries give substantially different second-harmonic generation. From Husu et al., 2012. 
reflectionless at both the fundamental frequency and the SH frequency (Kudyshev, Gabitov, and Maimistov, 2013). It was found that there is a critical value of the phase mismatch, below which the transformation of the FF into SH occurs monotonically, similar to the phase-matched case, but at a reduced rate. For phase mismatch above the critical value, the conversion occurs similar to the case of the phase-mismatched harmonic generation in the positive index medium, with low conversion efficiency. A similar tolerance to the phase mismatch was also found for the third-harmonic generation process that involves a backward fundamental frequency and a forward third-harmonic wave (Ostroukhova and Maimistov, 2012).

Another interesting regime of $\mathrm{SH}$ generation, which is discussed for the metamaterial structures, is when the phase velocities of the interacting waves vanish. In particular, it was demonstrated for the specially designed dual band composite right-left-handed transmission lines (see Sec. III.D) that it is possible to achieve such a regime (Somerville, Powell, and Shadrivov, 2011).

Using the freedom of metamaterial engineering, it was suggested (Gorkunov, Shadrivov, and Kivshar, 2006) that it is possible to enhance the nonlinear interaction by using double resonant metamaterials. This approach utilizes the result that the effective nonlinear susceptibility is proportional to the product of linear susceptibilities at the interacting frequencies. As a result, a metamaterial containing resonators of two types, with resonant frequencies differing by a factor of 2 , can lead to a significant enhancement of the second-harmonic generation. This enhancement was later demonstrated in microwave experiments (Kanazawa et al., 2011; Nakanishi, Tamayama, and Kitano, 2012). Note that the use of double resonance is also popular in photonics (Veronis and Fan, 2009; Bi et al., 2012).

In optics, most metamaterials have one or a few layers with metallic inclusions, and their properties are often dominated by surface plasmon polaritons, or localized plasmons when the metamaterial structure contains individual metallic nanoparticles. Rather than the nonlinear response of dielectrics, in plasmonic structures the nonlinearity is dominated by the response of free electrons in metals. The first experimental demonstration of second-harmonic generation for pulses reflected from a silver mirror was reported as early as 1965 (Brown, Parks, and Sleeper, 1965). Using structured metallic surfaces one can achieve substantial enhancement of secondharmonic generation (Wokaun et al., 1981; Tuovinen et al., 2002; Klein et al., 2006; E. Kim et al., 2008; Niesler et al., 2009; Wang et al., 2009; Husu et al., 2012). In particular, the efficiency of the SHG process depends on the symmetry of the nanostructures (Husu et al., 2012), and a seemingly small difference in the arrangement of the nanoparticles can lead to a dramatic change in the second-harmonic generation; see Figs. 12(b) and 12(c). At the same time, with an appropriate combination of centro-symmetric and non-centro-symmetric arrays (Czaplicki et al., 2013), SHG can be dramatically enhanced via plasmonic interactions.

Renewed interest in second-harmonic generation by metallic structures has also stimulated new theoretical and numerical studies in the area (Zeng et al., 2009; Biris and Panoiu, 2010; Scalora et al., 2010; de Ceglia et al., 2011), including the analysis of boosted SHG generation in ENZ media (de Ceglia et al., 2013).
Hyperbolic metamaterials also offer curious implications for second-harmonic generation: for example, in layered media a generated signal can be trapped, phase locked under the cone of a pump (de Ceglia et al., 2014).

The numerical solution of classical hydrodynamic equations for free electrons in metals along with finite-difference time-domain simulations of Maxwell's equations (Zeng et al., 2009) gave results for the generated second harmonics which are in good agreement with earlier experiments.

Third-harmonic generation and higher-order effects were also reported in microwave (Shadrivov et al., 2008b) and optical ranges (E. Kim et al., 2008; Scalora et al., 2010; Reinhold et al., 2012; Vincenti et al., 2012).

\section{Parametric amplification and loss compensation}

Parametric amplification is the process of amplifying a signal wave by using a high-power pump from which energy is transferred to the signal via nonlinear interaction. Since metamaterials typically rely on using metallic or high-index materials, significant attenuation of electromagnetic waves due to dissipation is often encountered. For this reason, parametric amplification was suggested as a means of compensating losses in negative-index materials (Kozyrev and van der Weide, 2005; Popov and Shalaev, 2006a), with demonstrations mostly performed in the microwave range, including transmission lines (Sec. III.D), with a patent pending (Kozyrev and van der Weide, 2006).

Along with the general theoretical discussion relevant for negatively refracting (Popov and Shalaev, 2006b) or lowindex (Feng and Halterman, 2008) media, specific setups considered for parametric amplification include transmission lines (Kozyrev, Kim, and van der Weide, 2006; Powell, Shadrivov, and Kivshar, 2009a), magnetoinductive arrays (Sydoruk, Kalinin, and Shamonina, 2007; Sydoruk, Shamonina, and Solymar, 2007) and ring resonators (Syms, Solymar, and Young, 2008) (see Fig. 13), bulk SRR structures (Gorkunov, Shadrivov, and Kivshar, 2006), and cavities (de Ceglia et al., 2007). Adopting a nonlinear loss compensation in the optical range is more challenging (Popov, Myslivets, and Shalaev, 2009).

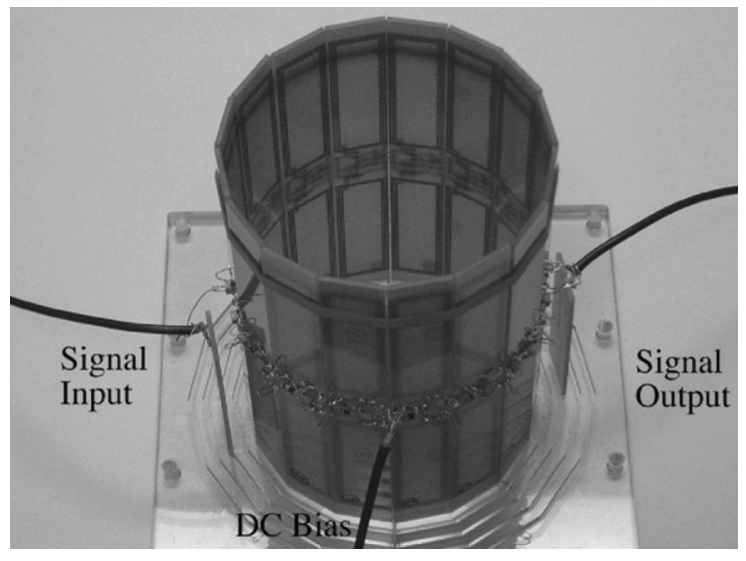

FIG. 13. Fabricated magnetoinductive ring resonator for the experimental observation of parametric amplification. From Syms, Solymar, and Young, 2008. 
While most studies rely on three-wave parametric processes, Popov et al. (2007) discussed compensation of losses by resonant optical parametric amplification with four-wave mixing, considering strongly absorbing composite metamaterials with a negative refractive index at the frequency of the signal, and a positive index for all other coupled waves. An important distinction of parametric amplification in metamaterials with negative refraction is that the pump wave energy should propagate in the direction opposite to that of the signal wave.

We note here that the applicability of assigning the opposite sign to the imaginary part of the effective parameters in order to account for compensated dissipation and gain should be taken with care (Zyablovsky et al., 2011) and checked against instability and lasing.

\section{Phase matching}

As pointed out earlier (Lapine and Gorkunov, 2004), the magnitude of nonlinear modulation in metamaterials can be quite strong, particularly in the microwave frequency range, so that a nonlinear contribution easily reaches a substantial fraction of the linear signal. This implies that phase-matching conditions, crucial for parametric processes and harmonic generation, may be relaxed under certain conditions, as a sufficient signal can be generated over a short interaction distance (one-wavelength slabs, for example), so that an accumulation of phase mismatch does not play such a significant role as in conventional nonlinear optics.

However, the particularly strong nonlinearity required for such situations is not universally available, so the problem of phase matching often has to be solved. Thanks to the advantages of metamaterials, this is easy to achieve as we have remarkable freedom in metamaterial design, which provides a route to dispersion engineering or built-in quasiphase matching.

The earliest approach to address phase matching via a specific design was relying on metamaterial arrays with dual resonances (Sydoruk et al., 2005), assembled as two subsystems of resonators with different resonant frequencies. Such metamaterials demonstrate two clearly separated resonance peaks; by an appropriate choice of the two resonances (Fig. 14), it is possible to design a dispersion curve where, e.g., the second harmonic $(2 \omega)$ has exactly double $(2 k)$ the wave vector of the fundamental wave $(\omega, k)$, automatically fulfilling phase requirements in the entire volume of the metamaterial sample (Gorkunov, Shadrivov, and Kivshar, 2006; Sydoruk et al., 2006).

Alternative options for phase matching are offered with the harmonic generation in reflection, available in nonlinear metamaterials with negative refraction (Agranovich et al., 2004; Shadrivov, Zharov, and Kivshar, 2006) — the so-called nonlinear-optical mirror. It is also possible to achieve automatic phase matching in metamaterial arrays, where the parameters can be adjusted so as to achieve matching conditions on a single dispersion curve (Sydoruk, Kalinin, and Shamonina, 2007).

Then, dispersion engineering, owing to unique linear properties of metamaterials, may play a significant role for phase matching, particularly if the effective wavelength in
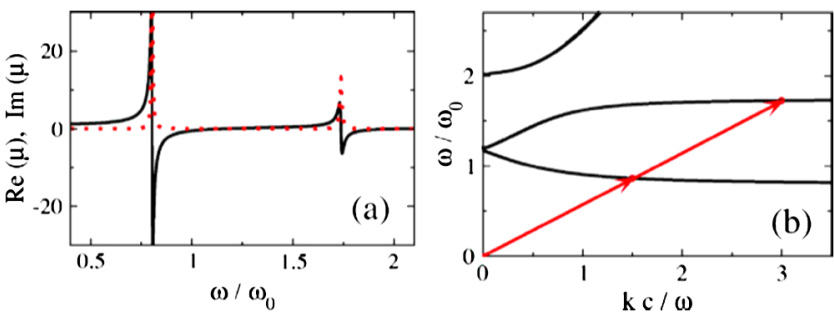

FIG. 14 (color online). Dual resonance and the corresponding dispersion diagram in binary metamaterials, allowing for SHG phase matching. From Gorkunov, Shadrivov, and Kivshar, 2006.

the medium becomes extremely large so that no phase is accumulated over the interaction length. This has been analyzed in transmission lines, between two waves with zero phase velocities (Somerville, Powell, and Shadrivov, 2011), and in a fishnet structure (Suchowski et al., 2013) imitating a close to zero refractive index.

A further advantage with regard to phase matching, specific to metamaterials with negative refraction, is related to the fact that in such a case monotonic generation of harmonics is quite robust against mismatch, as was shown for second(Kudyshev, Gabitov, and Maimistov, 2013) and thirdharmonic (Ostroukhova and Maimistov, 2012) generation, and apparently this is a general feature of parametric processes associated with a negative index.

Phase-locking phenomena and pulse trapping were analyzed (Roppo et al., 2007) for metamaterials with negative refraction, where an anomalous phase-matched harmonic was predicted to exist under certain parameters even in the absence of classical phase matching. In this process, a part of the harmonic is
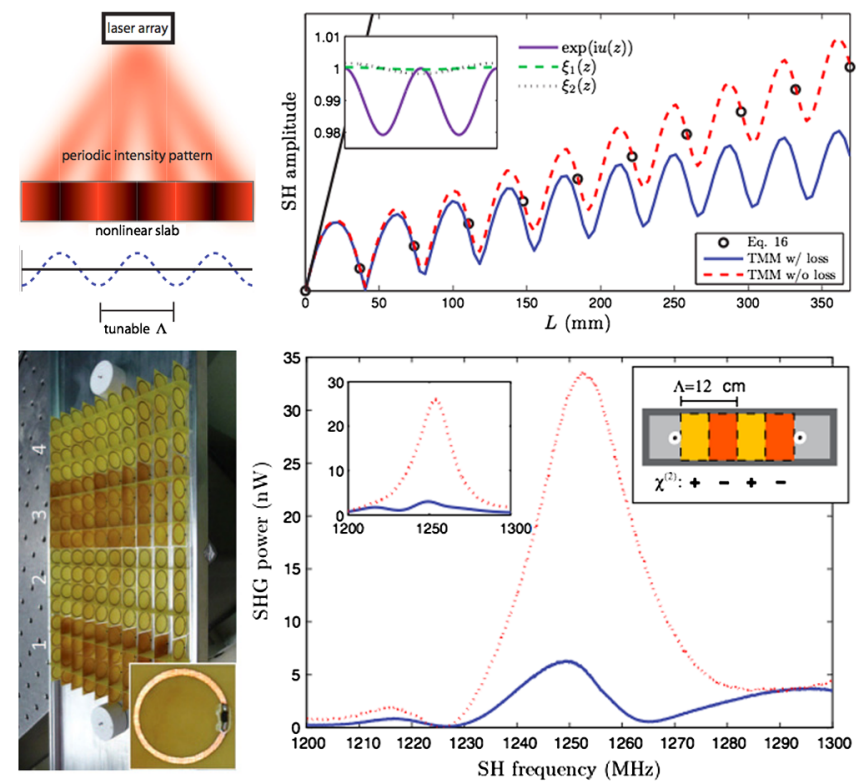

FIG. 15 (color online). Implementation of the quasiphase matching. Top: Periodic polling of the resonant frequency by illuminating the array of resonators with photosensitive inclusions with a specific light pattern, and the resulting SHG efficiency. From Rose and Smith, 2011. Bottom: Geometrically polled array of nonlinear SRRs, with the identical sections reoriented to achieve opposite bias in the varactors, and the resulting SHG efficiency. From Rose, Huang, and Smith, 2011. 
reflected and a part is trapped to propagate along with the fundamental wave. Interestingly, this behavior has no threshold power and no solitary nature; at the same time, it is dramatically sensitive to inhomogeneity in the nonlinear parameters and therefore might be difficult to realize in practice.

An interesting technical approach to realize quasiphase matching in metamaterials was proposed by Rose and Smith (2011): they designed split-ring resonators embedded in a semiconductor with the gaps filled with silicon. The photosensitivity of silicon inclusions was then used to tune the resonance of the meta-atoms by imposing a spatially periodic intensity pattern of laser illumination, adjusted to modulate the resonance strength to achieve phase matching via periodic perturbation (Fig. 15, top).

The ease of metamaterial design for microwaves also allows direct application of standard phase-matching principles, such as periodic poling. Rose, Huang, and Smith (2011) used identical sections of resonators, which were periodically reoriented in space, with the difference between the orientations determined by the direction of the possible current flow through the varactors loading the resonators (Fig. 15, bottom). A remarkable increase in SHG efficiency was observed in reoriented structures compared to the uniform ones, even though the total length of the metamaterial slab amounted to 16 unit cells in the direction of propagation.

\section{Phase conjugation}

Phase conjugation is a specific four-wave mixing process which aims to compensate any distortion of a signal propagating through a medium, by effectively reversing the wave front in the reflected signal. Admittedly the earliest approach to implement phase conjugation in artificial media was reported long before the metamaterial research had started: Kalinin and Shtykov (1990) proposed to use a medium of randomly distributed small dipoles loaded with varactor diodes. Their conclusions, however, were not optimistic as they expected a relatively high level of dissipation in the system.

Later on, Malyuskin, Fusco, and Schuchinsky (2006a) provided an in-depth analysis of the surface phase conjugation in periodic 2D arrays of nonlinearly loaded dipole antennas (Fig. 16), formulating their theory in terms of Pocklington's equation in both time and frequency domains, and considering the cases of quadratic and cubic nonlinearity. They also supported their analysis with numerical calculations, retrieving phase-conjugated signals in reflection and transmission with a single-layer array, for a double frequency pump in the case of quadratic nonlinearity, and a traditional fundamental frequency pump for cubic nonlinearity. Importantly, in the latter case, they found conditions to eliminate specular reflection. The effect of array spacing was also investigated, resulting in a set of practical recommendations. As a follow-up, a possibility to construct a lens based on a phase-conjugating frequency-selective surface was explored (Malyuskin, Fusco, and Schuchinsky, 2006b).

More recently, a few experimental results were reported attempting to observe phase conjugation in various metamaterial-inspired structures in the microwave (Katko et al., 2010; Katko, Shvets, and Cummer, 2012) and optical (Harutyunyan, Beams, and Novotny, 2013) ranges.

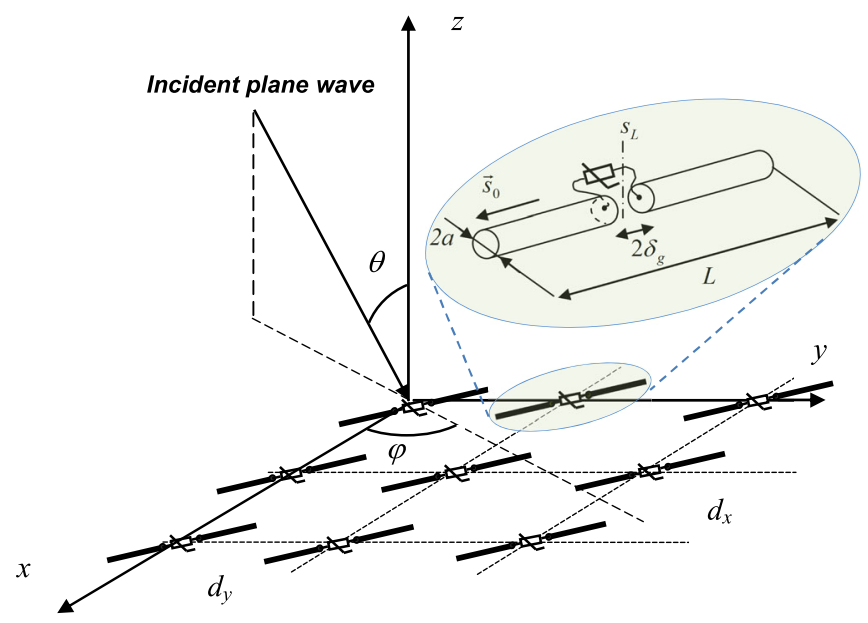

FIG. 16 (color online). Surface array of nonlinearly loaded dipoles, analyzed for microwave phase conjugation (Malyuskin, Fusco, and Schuchinsky, 2006a): A general geometry of the setup, with the schematic of a dipole antenna with nonlinear load. From A. Schuchinsky.

Apart from the phase conjugation as such, four-wave mixing processes in nonlinear nanostructures have been reported to imitate negative refraction in planar systems (Palomba et al., 2012) or to provide high conversion efficiency when used with plasmonic nanoantennas (Maksymov, Miroshnichenko, and Kivshar, 2013).

\section{Stimulated Raman scattering}

Another specific process which depends on the third-order nonlinear susceptibility is the scattering of propagating waves by optical phonons, known as Raman scattering. In metamaterials, apart from the likely requirement to consider the corresponding phenomena at lower frequencies than optical ones, some peculiarities of stimulated Raman scattering can be expected in cases where negative refraction is involved. This situation was briefly analyzed by Agranovich et al. (2004) and later on addressed by Maimistov and Gabitov (2007). In these papers, it was assumed that negative refraction is available only at the frequency of the Stokes wave, while the pump and the anti-Stokes components are in the range of normal positive refraction. As expected, in this case the Stokes wave would experience an exponential gain in the direction of pump propagation, opposite to the wave vector of the Stokes wave itself (Agranovich et al., 2004). The equations applicable for Raman amplification and spike generation in the presence of negative refraction were also derived (Maimistov and Gabitov, 2007), while more recently a scheme for coherent wave mixing enhancement based on stimulated Raman scattering was discussed (Popov et al., 2012). Experimentally, Raman enhancement was claimed (Ayas et al., 2012) for structured plasmonic metamaterial-like surfaces composed of metals and semiconductors (Fig. 17).

The choice of having a negative index for the Stokes component is quite natural with the resonant origin of negative parameters, as it avoids severe complications with having the pump wave in the vicinity of the metamaterial resonance. However, alternative scenarios are also interesting and waiting 

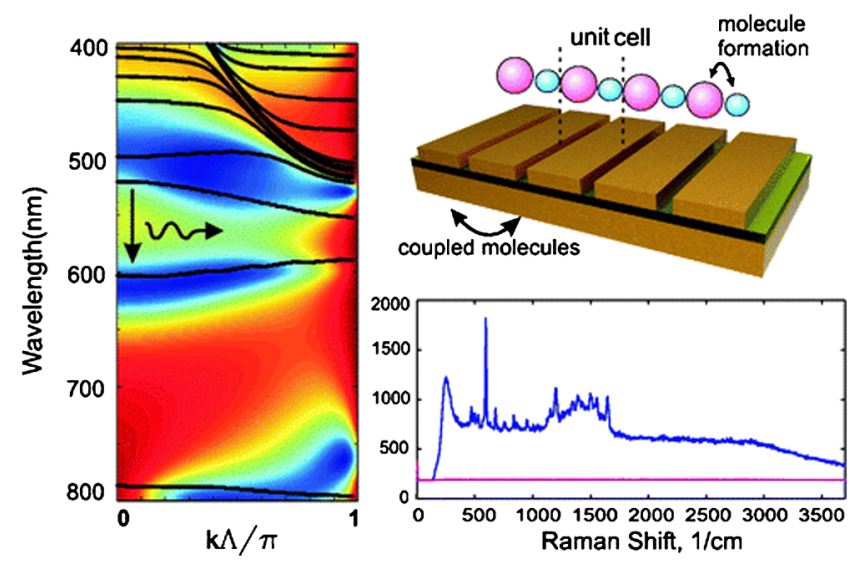

FIG. 17 (color online). Overall schematic of the Raman enhancement experiment using a photonic crystal composed of metal $(\mathrm{Ag})$ and dielectric $\left(\mathrm{Al}_{2} \mathrm{O}_{3}\right)$ layers. Band structure and collected Raman signal are shown. From Ayas et al., 2012.

to be analyzed. In particular, having the main waves in the region of zero index and the Stokes and anti-Stokes components closely around that range may lead to interesting observations.

The recent emergence of magnetoelastic metamaterials (Lapine et al., 2012) (see Sec. III.E) opens new opportunities for unusual coupling between electromagnetic and acoustic waves, so Mandelshtam-Brillouin and Raman scattering might return to the focus of research attention in the context of nonlinear metamaterials.

\section{Nonlinear subwavelength lenses}

One of the famous applications of metamaterials with negative refractive index is their use for a superlens (Pendry, 2000), which offers subwavelength focusing along with evanescent fields enhancement. An interesting improvement of superresolution was suggested by Zharov et al. (2005) with the use of quadratic nonlinearity, where a microwave superlens, assembled with nonlinear SRRs, is designed to be opaque for the fundamental while being negatively refracting for the second harmonic. This device provides a secondharmonic image whereas the original source could be screened. A similar effect was reported to occur when a negatively refracting linear layer is combined with a conventional nonlinear slab (Husakou and Herrmann, 2006).

The idea of nonlinear lensing was further theoretically explored for the optical range (Ciraci and Centeno, 2009), while experimental attempts were reported for microwave frequencies (Wang et al., 2011). Nonlinear imaging was also suggested with hyperbolic metamaterials (de Ceglia et al., 2014), with the second-harmonic signal generated from a pair of sources resolved, while the sources are indistinguishable at the fundamental frequency.

The impact of nonlinearities on the focusing performance of Lüneburg (gradient) lenses was also considered (Gao et al., 2011; Mattheakis, Tsironis, and Kovanis, 2012).

\section{Surface effects}

\section{Nonlinear surface waves}

Negative-index metamaterials attracted significant interest for waveguiding applications. Remarkably, the interface between positive-index dielectrics and metamaterials can support localized surface waves (Ruppin, 2000; Shadrivov et al., 2004). TM-polarized waves similar to surface plasmon polaritons are supported when the dielectric permittivity changes its sign at the interface. Additionally, negative-index materials can support TE-polarized surface polaritons, because magnetic permeability also changes its sign at the interface. Surface plasmon polaritons are widely used in sensing applications, and the ability to design structures supporting surface modes with desired properties, such as polarization, localization, and wavelength, is of paramount importance. Surface waves can also have an unwanted effect on the results of measurements, when scattering on defects may lead to the excitation of surface waves, and the measured spectra will contain contributions from surface waves.

Surface waves can be either forward or backward, meaning that their phase fronts propagate either with or against the energy flow, respectively (Shadrivov et al., 2004). Such behavior is possible because the energy flows in negativeindex materials and dielectrics are in opposite directions [see Fig. 18(a)], and their balance determines the direction of the total energy flow. To add one more possibility for manipulating the properties of surface guided waves, one may consider that the materials forming the interface are nonlinear (Shadrivov et al., 2004; Boardman et al., 2005; Darmanyan, Nevière, and Zakhidov, 2005; Boardman and Egan, 2009; Xu et al., 2009; Dai et al., 2010). In particular, this will allow the dispersion of the waves to be controlled (Shadrivov et al., 2004; Boardman et al., 2005), so that the type of the waves can be switched between forward and backward by adjusting the wave intensity; see Fig. 18(b). In other words, such tuning can allow changing the sign of the group velocity. In a
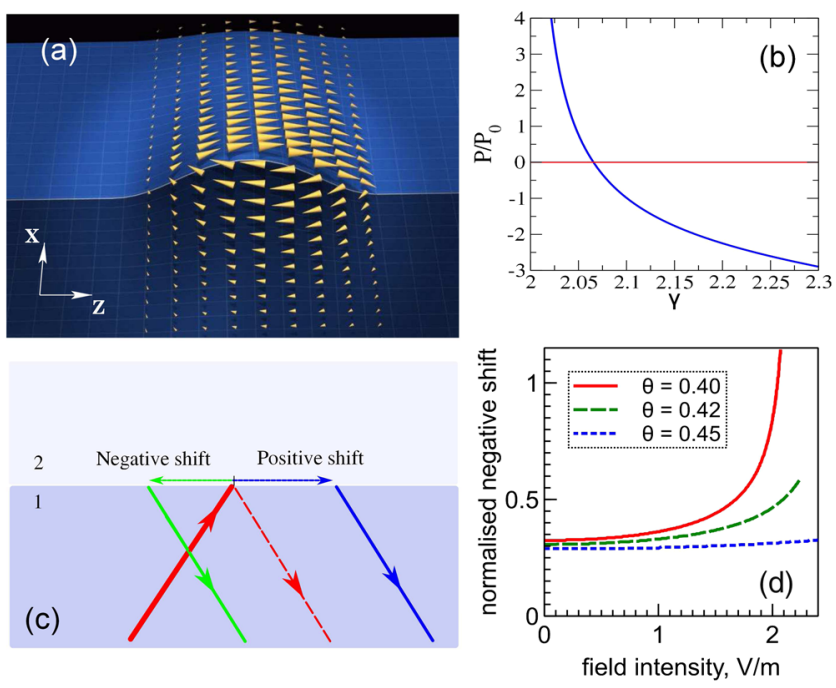

FIG. 18 (color online). (a) Energy flow diagram in a pulse propagating along the surface between a negative-index metamaterial and a positive-index dielectric. From Shadrivov et al., 2004. (b) Example of the normalized energy flow in the surface mode as a function of the wave number. From Shadrivov et al., 2004. (c) Schematic of negative and positive Goos-Hänchen shifts at the reflection from the interface between two media. (d) Dependence of the nonlinear Goos-Hänchen shift on the incident field intensity at various incident angles. 
particular point, when the group velocity vanishes, the energy flowing in the negative-index medium is compensated by that in the dielectric. Nonlinear change of the dispersion is also crucially important for the propagating temporal surface plasmon solitons (Shadrivov et al., 2004), the spreading of which is determined by the wave dispersion.

Another type of nonlinear surface waves is related to the nonlinear Tamm state (Entezar et al., 2008; Iorsh et al., 2012). Such waves are localized due to the band gap of the periodic structure and can exist at surfaces which do not support linear surface modes (Iorsh et al., 2012).

\section{Goos-Hänchen shift at a nonlinear interface}

A beam reflected from an interface between two media experiences a lateral displacement from its position predicted by geometric optics, because each of its plane-wave components acquires a different phase shift. For a beam reflected from an interface, the lateral shift is called the Goos-Hänchen effect and it is usually much smaller than the beam width. However, larger beam shifts may occur in either layered structures supporting surface waves, which are able to transfer energy along the interface, or at nonlinear interfaces, where the higher power of the incident beam may result in nonlinear surface wave excitation. Surface waves are not excited at a single interface between linear media, because the phasematching condition of the incident (propagating) and surface (localized) waves is not satisfied. The Goos-Hänchen shift can be either positive or negative [see schematics in Fig. 18(c)] depending on the type of the wave excited. In particular, it was predicted that a negative beam shift can be observed at the interface between linear dielectric and nonlinear negativeindex material: due to the nonlinearity, the effect depends on the intensity of the incident wave, as shown in Fig. 18(d) (Zhang, Chen, and Liang, 2008).

In the Otto configuration for the excitation of nonlinear surface waves it was shown that the beam shift becomes bistable (X. Chen et al., 2010) as a result of the resonant excitation of the surface waves. It was shown that wave intensity affects the resonant phase-matching condition for the wave excitation, thus providing a bistable response for the angle of incidence close to the resonance condition. Additionally, the Goos-Hänchen shift affects the group delay of the pulses scattered on finite slabs of negative-index metamaterial (Ilic et al., 2011).

\section{Nonlinear guided waves and solitons}

Guided waves with properties depending on the wave intensity represent a separate area of research. Nonlinear effects may not only change the properties of guided modes (Shadrivov, 2004) and introduce symmetry breaking effects, but they may also lead to the creation of new families of waves, such as spatial or temporal solitons. Spatial solitons are electromagnetic beams, whose diffraction is arrested by the nonlinear self-focusing, while temporal solitons are pulses whose dispersion broadening is compensated by nonlinear self-action effects (Kivshar and Agrawal, 2003). Such waves are actively studied in dielectric photonics and the emergence of metamaterials has added a new twist to the topic. While this particular branch of soliton physics is almost purely theoretical at the moment because of the complexity of manufacturing nonlinear-optical metamaterials and typically high dissipation, the diversity of predicted effects is quite extensive owing to the nontrivial linear and nonlinear properties of metamaterials. Most of the experiments were performed with left-handed transmission lines (Sec. III.D).

The models employed for the study of the nonlinear guided waves in metamaterials can be divided into two major categories. The first is a discrete approach, treating metamaterial as an array of coupled nonlinear resonators (Lazarides, Eleftheriou, and Tsironis, 2006; Shadrivov et al., 2006; Eleftheriou, Lazarides, and Tsironis, 2008; Lazarides, Tsironis, and Kivshar, 2008; Tsurumi, 2008; Cui et al., 2009b, 2010; W. Zhang et al., 2010; English et al., 2011). The second approach assumes that metamaterial is a homogeneous effective medium with specific dielectric permittivity, magnetic permeability, as well as nonlinear response (Boardman et al., 2005, 2008, 2010; Kourakis and Shukla, 2005; Lazarides and Tsironis, 2005; Scalora et al., 2005, 2006, 2007; Shadrivov and Kivshar, 2005; Zharova et al., 2005; Marklund, Shukla, and Stenflo, 2006; Banerjee and Nehmetallah, 2007; Kourakis, Lazarides, and Tsironis, 2007; Wen et al., 2007; D'Aguanno, Mattiucci, and Bloemer, 2008; Gelens et al., 2008; Hu et al., 2008; Yomba, 2008; Belicev et al., 2009; Cui et al., 2009a; Kazantseva, Maimistov, and Ozhenko, 2009; Kildishev et al., 2009; Kockaert et al., 2009; Martin and Hoyuelos, 2009; Tankeyev et al., 2009; Torner and Kartashov, 2009; Chen and Malomed, 2010; Ciattoni, Rizza, and Palange, 2010b; Gabitov, Kennedy, and Maimistov, 2010; Joseph and Porsezian, 2010; Li, Yang, and Xu, 2010; Skarka, Aleksić, and Berezhiani, 2010; Zhang, Wen, Xiang, Wang, and Luo, 2010; Du et al., 2011; Sarma, 2011; Yang and Zhang, 2011).

\section{Pulse propagation}

Solitons and pulse propagation in metamaterials attracted significant interest in the research community. Temporal solitons are predicted for metamaterials with resonant nonlinearity (Zharova et al., 2005), saturable nonlinearity (Belicev et al., 2009; Torner and Kartashov, 2009), quadratic nonlinearity (Scalora et al., 2006, 2007), cubic-quintic nonlinearity (Yang and Zhang, 2011), and cubic nonlinearity (Kourakis and Shukla, 2005; Lazarides and Tsironis, 2005; Scalora et al., 2005; Lazarides, Eleftheriou, and Tsironis, 2006; Marklund, Shukla, and Stenflo, 2006; Kourakis, Lazarides, and Tsironis, 2007; D'Aguanno, Mattiucci, and Bloemer, 2008; Eleftheriou, Lazarides, and Tsironis, 2008; Gelens et al., 2008; Eleftheriou et al., 2009; Kockaert et al., 2009; Tankeyev et al., 2009; Joseph and Porsezian, 2010; Martin and Hoyuelos, 2010; Sarma, 2011).

Metamaterials are strongly affected by dissipation, which should not be neglected in realistic models. As a result, stable temporal solitons do not exist in a pure form, but instead soliton-like pulses or dissipative solitons can form due to the presence of nonlinearity. As an example, soliton-like pulses can be generated by continuous waves in metamaterials with resonant nonlinearity (Zharova et al., 2005). Similar solitonlike pulses were experimentally observed in a composite righthanded or left-handed transmission line (Kozyrev and van der Weide, 2007). 
It was demonstrated experimentally that a novel mechanism of soliton generation can occur in nonlinear active transmission line metamaterial rings (Kozyrev, Shadrivov, and Kivshar, 2014). The structure consisting of a ring resonator formed by a microwave amplifier loaded with a nonlinear lefthanded transmission line can generate envelope backwardwave solitons (Fig. 19). This structure is much simpler than those which were used previously for soliton generation, and it does not require any high-frequency sources. The experimentally measured spatiotemporal dynamics of soliton generation in the system present a proof of the backward nature of the generated solitons, where it can be clearly seen that the pulse envelope propagates in the opposite direction to the phase fronts.

Directional couplers containing both positive- and negativeindex materials have been predicted to exhibit a threshold for soliton generation (Ryzhov and Maimistov, 2012). In particular, it was numerically shown that a pulse launched in the nonlinear positive-index arm of a coupler is emitted backward from the negative-index arm in the linear regime, while for sufficient power levels it can form a soliton and propagate in the positive direction (Ryzhov and Maimistov, 2012). Further theoretical studies (Kudryashov, Maimistov, and Sinelshchikov, 2012) demonstrated the existence of both nonlinear periodic and localized types of solution in such type of coupler.

In discrete systems, where metamaterials are explicitly treated as arrays of interacting resonators, various soliton solutions have been predicted, including bright and dark solitons (Kourakis, Lazarides, and Tsironis, 2007), domain walls (Shadrivov et al., 2006), breathers (Lazarides, Eleftheriou, and Tsironis, 2006), as well as surface breathers (Lazarides, Tsironis, and Kivshar, 2008). Most common discrete models assume that each resonator $n$ interacts only with its neighbors. For a one-dimensional chain, the equation describing currents $I_{n}$ can be written as (Lazarides, Eleftheriou, and Tsironis, 2006; Shadrivov et al., 2006)

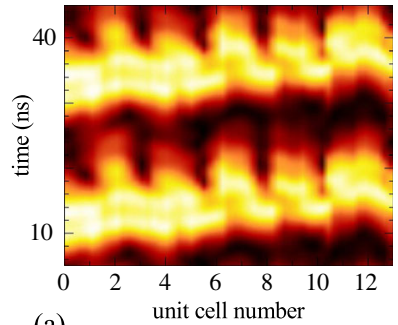

(a)

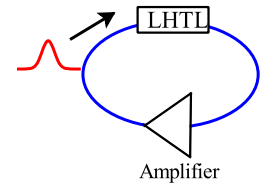

One pulse in the loop

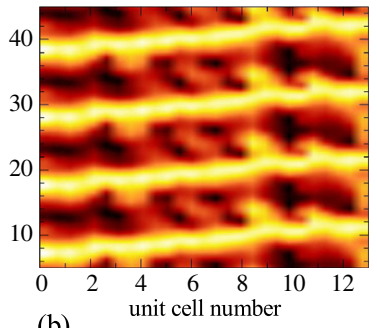

(b)

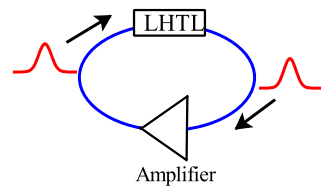

Two pulses in the loop
FIG. 19 (color online). Spatiotemporal dynamics of solitons inside the left-handed transmission line. Shown are the regimes when (a) one or (b) two solitons are generated in the rings (Kozyrev, Shadrivov, and Kivshar, 2014).

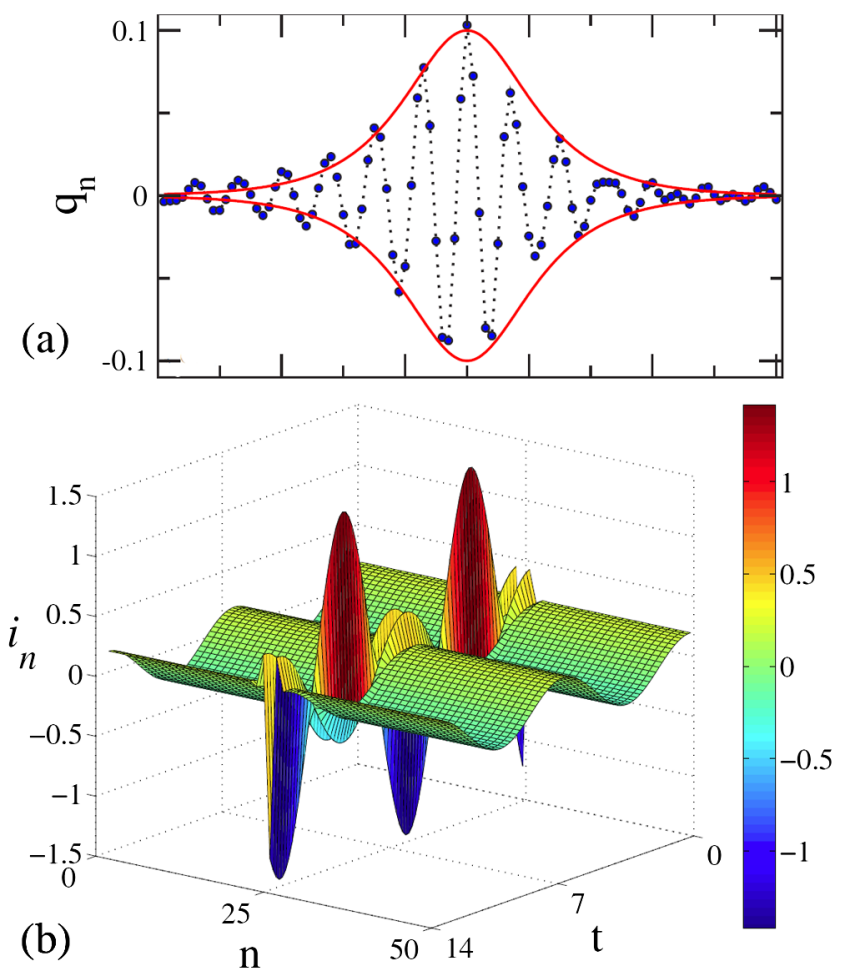

FIG. 20 (color online). (a) Temporal soliton (Kourakis, Lazarides, and Tsironis, 2007) and (b) a breather (Lazarides, Eleftheriou, and Tsironis, 2006) in the discrete array of split-ring resonators.

$$
L \frac{d I_{n}}{d t}+R I_{n}+f\left(Q_{n}\right)=M\left(\frac{d I_{n-1}}{d t}+\frac{d I_{n+1}}{d t}\right)+\mathcal{E},
$$

where $L$ is self-inductance of the resonator, $R$ is resistance, $f\left(Q_{n}\right)$ is the voltage on the nonlinear capacitance of the resonator, $Q_{n}$ is the charge on the capacitor, $M$ is the mutual interaction term, and $\mathcal{E}$ is the excitation. Depending on the parameters and the type of nonlinearity, Eq. (4) supports dissipative solitons shown in Fig. 20(a) or discrete breathers shown in Fig. 20(b).

In connection with nonlinear pulse propagation, self-focusing of the propagating beams (Kourakis, Lazarides, and Tsironis, 2007; Hu and Zhuo, 2009) as well as self-trapping phenomena and the formation of hot spots in active nonlinear media (Korotkevich et al., 2013) was theoretically discussed.

\section{Spatial solitons}

Spatial solitons are beams which have their diffractive broadening compensated by nonlinear self-focusing (Kivshar and Agrawal, 2003). In the case of Kerr-type nonlinearity, when the nonlinear correction to the dielectric constant is proportional to the intensity of the electric field, the nonlinear Schrödinger equation governing the propagation of paraxial beams leads to one-dimensional sech-type soliton solutions, which do not change along the propagation direction. Metamaterials, however, are predicted to have a more complex resonant nonlinear response (Zharov, Shadrivov, and Kivshar, 2003), where the response of the material is a bistable function of the magnetic field of the wave. For resonant nonlinearities, spatial solitons can be found by using a phase diagram method. In particular, various families of single-hump and 
multihump solitons were found by Shadrivov and Kivshar (2005). Because of the bistable nature of such nonlinearity, solitons may appear due to the formation of self-induced waveguides with properties corresponding to different branches of bistable characteristics.

For relatively low powers, when the nonlinear response is already pronounced, but when it is not multistable, the nonlinearity of the metamaterials can be reduced to a cubic or cubic-quintic nonlinearity. In this case, the equations governing the wave propagation reduce to the generalized nonlinear Schrödinger equation (NLSE). In the case of dual nonlinear behavior, when both electric and magnetic responses are nonlinear, i.e., $\varepsilon=\varepsilon_{1}+\varepsilon_{3}|E|^{2}$ and $\mu=$ $\mu_{1}+\mu_{3}|H|^{2}$, there is a number of works deriving coupled NLSEs for electric and magnetic fields, and claiming their irreducibility. We believe that this is not correct, and in the case of such dual nonlinearity, the electric and magnetic fields in plane waves are related via wave impedance. In particular, as shown by Boardman et al. (2010), the TE-polarized waves are governed by a NLSE of the following form:

$$
2 i k_{z} \frac{\partial E_{x}}{\partial z}+\frac{\partial^{2} E_{x}}{\partial x^{2}}=-\frac{\omega^{2}}{c^{2}}\left[\mu_{1} \varepsilon_{3}+\varepsilon_{1} \mu_{3} \frac{\left|\varepsilon_{1}\right|}{\left|\mu_{1}\right|}\right]\left|E_{x}^{2}\right| E_{x},
$$

where $k_{z}$ is the propagation constant, $\omega$ is the angular frequency, and $c$ is the speed of light in a vacuum. This equation neglects nonlinear diffraction and higher-order nonlinear effects, which are discussed in detail by Boardman et al. (2010). In particular, stationary solitons shown in Fig. 21(a) start diverging when the nonlinear diffraction becomes significant, as shown in Fig. 21(b).

Another interesting phenomenon related to spatial solitons is called the "soliton lens" (Boardman et al., 2005). The idea is that if the soliton enters a linear dielectric, it starts diffracting. Adding a layer of negative-index material allows compensation of this diffraction and restoration of the original soliton beam.

Arrays of subwavelength metal-dielectric layers are often analyzed in the context of metamaterials, prominent for hyperbolic dispersion, or subwavelength imaging. Narrow beams launched in such structures along the layers will diffract, coupling to the adjacent layers. However, Liu et al. (2007) predicted that the use of Kerr-type nonlinear dielectric layers in the structure leads to the formation of spatial solitons. Remarkably, such spatial solitons can be made narrower than the wavelength.
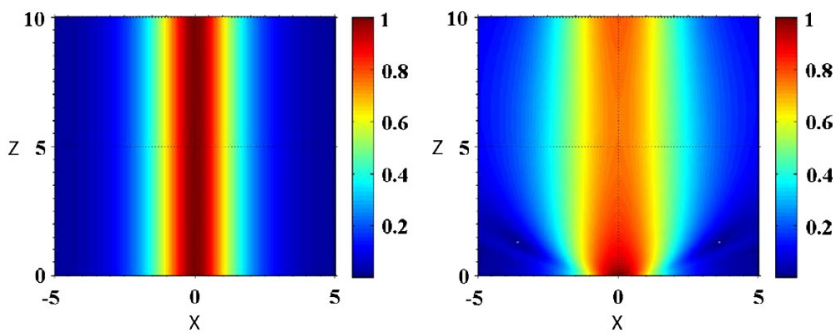

FIG. 21 (color online). Spatial solitons propagating in the nonlinear left-handed metamaterial: (left) without nonlinear diffraction and (right) with nonlinear diffraction. From Boardman et al., 2010.

\section{E. Discreteness effects}

As mentioned previously, the important building blocks of electromagnetic metamaterials are the SRRs or other types of subwavelength resonant elements which are arranged in one-, two-, or three-dimensional lattices. In general, the response of a metamaterial is not simply given by the sum of the responses of individual resonators, but also depends on the interaction between the resonators within the system (Gorkunov et al., 2002; Shamonina et al., 2002; Syms et al., 2005), which can be particularly sophisticated when the near-field interaction is involved (Hesmer et al., 2007; Powell et al., 2010). A standard theoretical approach for analyzing the properties of metamaterials is based on the effective medium approximation when the structure is treated as a homogeneous medium being characterized by effective macroscopic parameters. However, with finite size (Lapine, Jelinek, and Marqués, 2012), as well as with larger wave vectors, metamaterials demonstrate their discrete nature and strong nonlocal effects, so they should be described as arrays or lattices of resonant elements.

Discreteness effects are essential for parametric amplification in coupled magnetoinductive waveguides (Sydoruk, Shamonina, and Solymar, 2007). In that case phase-matching conditions (Sec. IV.B.3) can be satisfied by tailoring the dispersion characteristic of magnetoinductive waves, governed by discrete structure. A similar approach can be employed for parametric amplification of magnetoinductive waves in arrays (Sydoruk, Kalinin, and Shamonina, 2007).

Nonlinear physics of discrete metamaterials can be studied using coupled nonlinear equations describing nonlinear magnetoinductive waves (Lazarides, Eleftheriou, and Tsironis, 2006; Shadrivov et al., 2006). This approach was further generalized by Rosanov et al. (2011a, 2011b), taking into account both electric and magnetic near-field coupling between the neighboring sites.

The current $I_{\mathbf{r}}$ in a split-ring resonator located at the point $\mathbf{r}$ can be expressed through inductance $L$, resistance $R$, and capacitance $C$ of the resonator, as well as the mutual coupling terms (Shadrivov et al., 2006). When the capacitance of the resonator is nonlinear, i.e., it depends on the voltage $U$ across the gap of the resonator: $\left.C_{N L}=C_{0}+\left.\Delta C_{N L}\left(\mid U_{\mathbf{r}}\right)\right|^{2}\right)$, we can employ the slowly varying approximation and obtain the following equation for the normalized current in the split-ring resonator:

$$
i \frac{d \psi_{\mathbf{r}}}{d \tau}-\left(2 \Omega-i \gamma+\alpha\left|\psi_{\mathbf{r}}\right|^{2}\right) \psi_{\mathbf{r}}=S_{\mathbf{r}}+\sum_{\mathbf{j}} K_{\mathbf{j}} \psi_{\mathbf{j}},
$$

where dimensionless time, currents, and external electromotive force are $\tau, \psi_{\mathbf{r}}$, and $S_{\mathbf{r}}$, respectively, and the sum is performed over the nearest neighbors (Rosanov et al., 2011b). The interaction coefficients $K_{\mathbf{j}}$ crucially depend on the mutual position of the resonators, and the distributions of currents and charges in conductors (Hesmer et al., 2007; Powell et al., 2010).

The system of equations (6) describes a variety of nonlinear modes (Shadrivov et al., 2006; Rosanov et al., 2011a, 2011b), and one of the simplest nonlinear magnetoinductive waves is a switching wave (also called a domain wall or kink). A switching wave represents a transition from one uniform state to another one with a change in resonator number. Such 

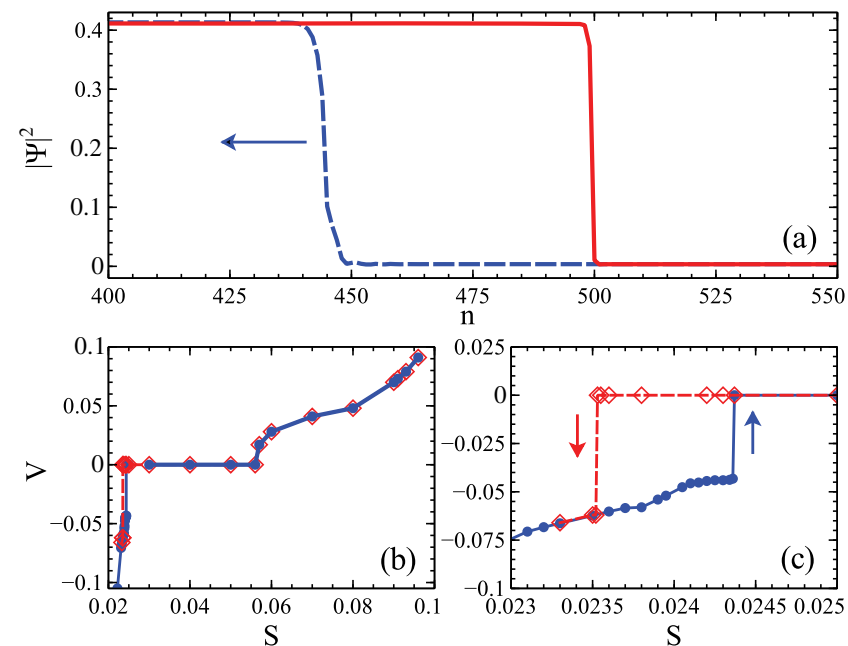

FIG. 22 (color online). (a) Distribution of magnetization for $S=0.024$. Standing (solid) and moving (dashed) domain walls. (b) Dependence of the velocity of the domain wall as a function of the external excitation $S$. (c) Close-up of the bistable region in (b), with arrows indicating directions of the jumps. From Rosanov et al., 2011b).

waves are initially excited by a nonuniform distribution of the external field, and they are supported by a uniform external excitation. The profiles of such waves are close to a step function, with typical domain wall structure shown in Fig. 22.

The same system of nonlinear equations describes spatially localized modes in the form of discrete solitons or discrete breathers (Tsitsas et al., 2010), as well as their states near the surface (Lazarides, Tsironis, and Kivshar, 2008; Eleftheriou et al., 2009). Such localized modes appear due to on-site nonlinearity and weak coupling among the individual elements. Such discrete single-breather and multibreather excitations, their mobility, and the magnetic properties were analyzed in the framework of the reduced model (6) (Rosanov et al., 2011a, 2011b) as well as a complete model (Lazarides, Eleftheriou, and Tsironis, 2006; Eleftheriou, Lazarides, and Tsironis, 2008). Such localized modes were shown to exist in both one- and two-dimensional lattices of SRRs. Figure 23 shows two examples of two-dimensional localized modes (discrete breathers) in an anisotropic lattice of SRRs of a planar geometry.

The above consideration can be generalized to threedimensional lattices of nonlinear subwavelength elements. Such structures can support entirely new classes of spatially

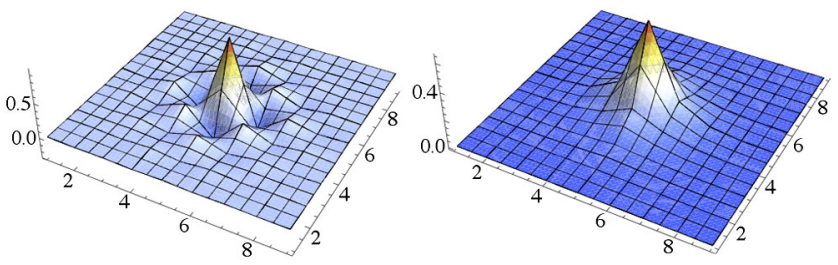

FIG. 23 (color online). Snapshots of two-dimensional dissipative single sites, bright discrete breathers taken at maximum amplitude, for anisotropic split-ring resonator lattices in planar geometry (Eleftheriou, Lazarides, and Tsironis, 2008). From N. Lazarides. localized modes: knotted solitons, which are stable selflocalized dissipative structures in the form of closed knotted chains (Rosanov et al., 2012); they demonstrated different topological types of stable knots for the subcritical coupling between resonators and instability-induced breaking of the chains for the supercritical coupling.

\section{F. Related topics}

While we endeavored to introduce almost every aspect of nonlinear metamaterials, a few related areas were not explicitly covered. For instance, we did not present the area of active metamaterials, which is becoming an important stand-alone topic and was recently briefly reviewed by Boardman et al. (2011). We also did not describe the large area of tunable metamaterials in its entirety, but addressed this topic only from a perspective of nonlinear properties as such. Nonlinear plasmomics, recently reviewed by Kauranen and Zayats (2012), was also touched on only briefly in our survey. Finally, we did not include the emerging area of nonlinear acoustic metamaterials, which seems to be increasingly appreciated, with several publications to date (Herbold and Nesterenko, 2007, 2013; Giordano, Palla, and Colombo, 2008; Manktelow, Leamy, and Ruzzene, 2011; Girchenko, Eremeyev, and Altenbach, 2012; Manktelow et al., 2013).

\section{CONCLUSIONS AND PERSPECTIVES}

We presented an overview of the rapid progress in the physics and applications of nonlinear metamaterials over the decade since their emergence in the literature. It is clear that the research area of nonlinear metamaterials has provided an impressive flow of publications which is yet to reach its peak and to provide a valuable overall impact on the fields of electromagnetics and optics.

The key outcomes of nonlinear metamaterials with regard to nonlinear electrodynamics in general, as well as for metamaterials development, can be summarized as follows:

- Demonstration of exceptionally high nonlinearities, sometimes with over $10 \%$ of nonlinear modulation being easily achievable, and diverse analysis of the resulting phenomena.

- Emergence of substantial nonlinearity in magnetic response, which is sourced from conventional electric features but translated to magnetic properties by virtue of metamaterial design.

- Offering a path toward dissipation compensation via nonlinear parametric processes.

- Analysis of a great range of unusual nonlinear phenomena, triggering wide academic interest and promising fruitful future applications.

At the same time, the accomplishments in this research area are still far from being exhausted, and we foresee the following challenges becoming the major driving points for future research:

- Development of consistent theoretical models for analyzing metamaterials with a strong spatial dispersion along with a strong nonlinearity. 
- Exploration of alternative possibilities for nonlinear feedback, with a particular emphasis on crossdisciplinary solutions and extra degrees of freedom.

- Optimization and improvement of the design of nonlinear metamaterials, targeting practical implementation and applications.

It is expected that the future success of the entire field of metamaterials will depend on their ability to bring novel solutions and approaches to existing photonics technologies. Future technologies should be capable of contributing substantially to both photonic integration and energy efficiency, allowing the breakthrough technologies exceeding the currently available bulk optical components, silicon photonics, and plasmonic circuits. It is believed that metamaterials will bring unique functionalities by allowing the engineering of the material parameters at the level of their elementary units (meta-atoms) creating a new paradigm of metadevices.

One of the important anticipated developments in this field is the demonstration of many of the nonlinear effects known in nonlinear physics and nonlinear optics such as nonlinear selfaction, parametric interactions, and frequency conversion, which will boost the development of various methods for achieving tunable, switchable, nonlinear, and sensing functionalities of metamaterials. The study of nonlinear effects in artificial media and engineering the nonlinear response of such media are crucially important for this progress.

In the context of photonic integration, metamaterials promise pathways for light that are impossible in normal materials, and offer new freedom in exploiting nonlinear processes. While advances in lithography now allow metamaterials to be fabricated at optical wavelengths, material and fabrication constraints have hampered progress. The key progress in this direction would be to fine-tune metamaterials by changing the properties of their material constituents. By incorporating nonlinear and tunable materials, it will be possible to create functional metamaterials that display sensitive tuning and novel or enhanced nonlinear behavior. These materials will ultimately provide the basis of a revolutionary platform for optical processing.

\section{ACKNOWLEDGMENTS}

We are indebted to David R. Smith, Martti Kauranen, Michael Scalora, Richard Syms, Andrei Maimistov, Maxim Gorkunov, and Alexey Ustinov who have read the original version of the manuscript and offered their valuable suggestions. We are also grateful to David A. Powell for his help in preparing the final version of this review. The work on this review was supported by the Australian Research Council (via CUDOS Centre of Excellence CE110001018, and Discovery Projects).

\section{REFERENCES}

Abb, M., B. Sepúlveda, H. Chong, and O. Muskens, 2012, J. Opt. 14, 114007.

Abdumalikov, A., O. Astafiev, A. Zagoskin, Y. Pashkin, Y. Nakamura, and J. Tsai, 2010, Phys. Rev. Lett. 104, 193601.

Agranovich, V. M., and Y. N. Gartstein, 2009, Metamaterials 3, 1.
Agranovich, V. M., Y.R. Shen, R.H. Baughman, and A. A. Zakhidov, 2004, Phys. Rev. B 69, 165112.

Akhmanov, S., B. Zdanov, N. Zheludev, A. Kovrigin, and V. Kuznetsov, 1979, JETP Lett. 29, 264.

Albooyeh, M., D. Morits, and C. R. Simovski, 2011, Metamaterials 5, 178.

Alitalo, P., S. Maslovski, and S. Tretyakov, 2006, J. Appl. Phys. 99, 124910.

Andryieuski, A. S., S. Ha, A. A. Sukhorukov, Y. S. Kivshar, and A. V. Lavrinenko, 2012, Phys. Rev. B 86, 035127.

Anlage, S., 2011, J. Opt. 13, 024001.

Argyropoulos, C., N. Estakhri, F. Monticone, and A. Alù, 2013, Opt. Express 21, 15037.

Astafiev, O., A. Abdumalikov, A. Zagoskin, Y. Pashkin, Y. Nakamura, and J. Tsai, 2010, Phys. Rev. Lett. 104, 183603.

Astafiev, O., A. Zagoskin, A. Abdumalikov, Y. Pashkin, T. Yamamoto, K. Inomata, Y. Nakamura, and J. Tsai, 2010, Science 327, 840.

Ayas, S., H. Güner, B. Türker, O. Ekiz, F. Dirisaglik, A. Okyay, and A. Dâna, 2012, ACS Nano 6, 6852.

Baena, J. D., L. Jelinek, R. Marqués, and M. Silveirinha, 2008, Phys. Rev. A 78, 013842.

Banerjee, P., and G. Nehmetallah, 2007, J. Opt. Soc. Am. B 24, A69.

Belicev, P., I. Ilic, V. Milanovic, J. Radovanovic, and L. Hadievski, 2009, Phys. Rev. A 80, 023821.

Belov, P. A., R. Marqués, S. I. Maslovski, I. S. Nefedov, M. Silveirinha, C. R. Simovski, and S. A. Tretyakov, 2003, Phys. Rev. B 67, 113103.

Belov, P. A., and C. R. Simovski, 2006, Phys. Rev. B 73, 045102.

Bi, Z.-F., A. Rodriguez, H. Hashemi, D. Duchesne, M. Loncar, K.-M. Wang, and S. Johnson, 2012, Opt. Express 20, 7526.

Biris, C., and N. Panoiu, 2010, Phys. Rev. B 81, 195102.

Bloembergen, N., and A. J. Sievers, 1970, Appl. Phys. Lett. 17, 483.

Boardman, A., and P. Egan, 2009, J. Opt. A 11, 114032.

Boardman, A., P. Egan, L. Velasco, and N. King, 2005, J. Opt. A 7, S57.

Boardman, A., V. Grimalsky, Y. Kivshar, S. Koshevaya, M. Lapine, N. Litchinitser, V. Malnev, M. Noginov, Y. Rapoport, and V. Shalaev, 2011, Laser and Photonics Reviews 5, 287.

Boardman, A., N. King, R. Mitchellthomas, V. Malnev, and Y. Rapoport, 2008, Metamaterials 2, 145.

Boardman, A., R. Mitchell-Thomas, N. King, and Y. Rapoport, 2010, Opt. Commun. 283, 1585.

Boulais, K., D. Rule, S. Simmons, F. Santiago, V. Gehman, K. Long, and A. Rayms-Keller, 2008, Appl. Phys. Lett. 93, 043518.

Boyd, R. W., 2003, Nonlinear Optics (Academic Press, San Diego).

Brown, F., R. E. Parks, and A. M. Sleeper, 1965, Phys. Rev. Lett. 14, 1029.

Butz, S., P. Jung, L. Filippenko, V. Koshelets, and A. Ustinov, 2013, Opt. Express 21, 22540.

Cai, W., and V. Shalaev, 2009, Optical Metamaterials: Fundamentals and Applications (Springer, New York).

Caloz, C., and T. Itoh, 2006, Electromagnetic Metamaterials: Transmission Line Theory and Microwave Applications (Wiley, $\mathrm{NJ})$.

Campione, S., D. de Ceglia, M. Vincenti, M. Scalora, and F. Capolino, 2013, Phys. Rev. B 87, 035120.

Canfield, B. K., H. Husu, J. Laukkanen, B. Bai, M. Kuittinen, J. Turunen, and M. Kauranen, 2007, Nano Lett. 7, 1251.

Capolino, F., 2009, Handbook on Metamaterials (CRC Press, Boca Raton, FL).

Carbonell, J., V. Boria, and D. Lippens, 2008, Microwave Opt. Technol. Lett. 50, 474. 
Cavalcanti, S., P. Brandao, A. Bruno-Alfonso, and L. Oliveira, 2014, Opt. Lett. 39, 178.

Centeno, E., and C. Ciraci, 2008, Phys. Rev. B 78, 235101.

Chen, H.-T., H. Yang, R. Singh, J. F. OHara, A. K. Azad, S. A. Trugman, Q. X. Jia, and A. J. Taylor, 2010, Phys. Rev. Lett. 105, 247402.

Chen, P., and B. Malomed, 2010, Opt. Commun. 283, 1598.

Chen, P.-Y., M. Farhat, and A. Alu, 2011, Phys. Rev. Lett. 106, 105503.

Chen, X., R.-R. Wei, M. Shen, Z.-F. Zhang, and C.-F. Li, 2010, Appl. Phys. B 101, 283.

Chowdhury, A., and J. Tataronis, 2008, Phys. Rev. Lett. 100, 153905.

Chowdhury, R. D., R. Singh, J. O'Hara, H.-T. Chen, A. Taylor, and A. Azad, 2011, Appl. Phys. Lett. 99, 231101.

Ciattoni, A., C. Rizza, and E. Palange, 2010a, Phys. Rev. A 81, 043839.

Ciattoni, A., C. Rizza, and E. Palange, 2010b, Opt. Express 18, 11911.

Ciraci, C., and E. Centeno, 2009, Phys. Rev. Lett. 103, 063901.

Coelho, Jr., A. G., A. S. B. Queiroz, M. G. da Silva, M. L. Lyra, and A. S. B. Sombra, 2013, Communications in Nonlinear Science and Numerical Simulation, Vol. 18, p. 1258.

Colestock, P. L., M. T. Reiten, and J. F. O'Hara, 2012, Metamaterials 6, 8 .

Cui, W., Y. Zhu, H. Li, and S. Liu, 2009a, Phys. Lett. A 374, 380.

Cui, W., Y. Zhu, H. Li, and S. Liu, 2009b, Phys. Rev. E 80, 036608.

Cui, W., Y. Zhu, H. Li, and S. Liu, 2010, Phys. Rev. E 81, 016604.

Czaplicki, R., H. Husu, R. Siikanen, J. Mäkitalo, M. Kauranen, J. Laukkanen, J. Lehtolahti, and M. Kuittinen, 2013, Phys. Rev. Lett. 110, 093902.

Czaplicki, R., M. Zdanowicz, K. Koskinen, J. Laukkanen, M. Kuittinen, and M. Kauranen, 2011, Opt. Express 19, 26866.

D’Aguanno, G., N. Mattiucci, and M. Bloemer, 2008, J. Opt. Soc. Am. B 25, 1236.

D’Aguanno, G., N. Mattiucci, M. Scalora, and M. Bloemer, 2006, Phys. Rev. E 74, 026608.

Dai, X., Y. Xiang, S. Wen, and D. Fan, 2009, J. Opt. Soc. Am. B 26, 564.

Dai, X., Y. Xiang, S. Wen, and D. Fan, 2010, Opt. Commun. 283, 1607.

Dani, K., Z. Ku, P. Upadhya, R. Prasankumar, A. Taylor, and S. Brueck, 2011, Opt. Express 19, 3973.

Darmanyan, S., M. Nevière, and A. Zakhidov, 2005, Phys. Rev. E 72, 036615.

de Ceglia, D., S. Campione, M. Vincenti, F. Capolino, and M. Scalora, 2013, Phys. Rev. B 87, 155140.

de Ceglia, D., G. D’Aguanno, N. Mattiucci, M. A. Vincenti, and M. Scalora, 2011, Opt. Lett. 36, 704.

de Ceglia, D., A. D. Orazio, M. de Sario, V. Petruzzelli, M. G. Cappeddu, M. J. Bloemer, and M. Scalora, 2007, Opt. Lett. 32, 265.

de Ceglia, D., M. A. Vincenti, S. Campione, F. Capolino, J. W. Haus, and M. Scalora, 2014, Phys. Rev. B 89, 075123.

Degiron, A., J. J. Mock, and D. R. Smith, 2007, Opt. Express 15, 1115.

de Lorenci, V., and J. Pereira, 2012, Phys. Rev. A 86, 013801.

Drachev, V., V. A. Podolskiy, and A. V. Kildishev, 2013, Opt. Express 21, 15048

Du, C., H. Chen, and S. Li, 2006, Phys. Rev. B 74, 113105.

Du, Y., Z. Zhou, H. Tian, and D. Liu, 2011, J. Opt. 13, 015201.
Eleftheriades, G. V., and K. G. Balmain, 2005, Negative-Refraction Metamaterials: Fundamental Principles and Applications (Wiley, NJ).

Eleftheriou, M., N. Lazarides, and G. Tsironis, 2008, Phys. Rev. E 77, 036608.

Eleftheriou, M., N. Lazarides, G. Tsironis, and Y. Kivshar, 2009, Phys. Rev. E 80, 017601.

English, L., S. Wheeler, Y. Shen, G. Veldes, N. Whitaker, P. Kevrekidis, and D. Frantzeskakis, 2011, Phys. Lett. A 375, 1242.

Entezar, S., A. Namdar, Z. Eyni, and H. Tajalli, 2008, Phys. Rev. A 78, 023816.

Fan, K., H. Hwang, M. Liu, A. Strikwerda, A. Sternbach, J. Zhang, X. Zhao, X. Zhang, K. Nelson, and R. Averitt, 2013, Phys. Rev. Lett. 110, 217404.

Fan, Y., J. Han, Z. Wei, C. Wu, Y. Cao, X. Yu, and H. Li, 2011, Appl. Phys. Lett. 98, 151903.

Fedotov, V. A., A. Tsiatmas, J. H. Shi, R. Buckingham, P. de Groot, Y. Chen, S. Wang, and N. I. Zheludev, 2010, Opt. Express 18, 9015.

Feise, M., I. Shadrivov, and Y. Kivshar, 2004, Appl. Phys. Lett. 85, 1451.

Feise, M., I. Shadrivov, and Y. Kivshar, 2005, Phys. Rev. E 71, 037602.

Feng, S., and K. Halterman, 2008, Phys. Rev. Lett. 100, 063901.

Gabitov, I., B. Kennedy, and A. Maimistov, 2010, IEEE J. Sel. Top. Quantum Electronics 16, 401.

Gabitov, I. R., R. A. Indik, N. M. Litchinitser, A. I. Maimistov, V. M. Shalaev, and J. E. Soneson, 2006, J. Opt. Soc. Am. B 23, 535.

Gao, H., S. Takahashi, L. Tian, and G. Barbastathis, 2011, Opt. Express 19, 2257.

Gelens, L., D. Gomila, G. van der Sande, J. Danckaert, P. Colet, and M. Matias, 2008, Phys. Rev. A 77, 033841.

Gelens, L., G. van der Sande, P. Tassin, M. Tlidi, P. Kockaert, D. Gomila, I. Veretennicoff, and J. Danckaert, 2007, Phys. Rev. A 75, 063812 .

Ginzburg, P., et al., 2013, Opt. Express 21, 14907.

Giordano, S., 2010, J. Electrost. 68, 227.

Giordano, S., P. Palla, and L. Colombo, 2008, Europhys. Lett. 83, 66 003.

Giordano, S., and W. Rocchia, 2005, J. Appl. Phys. 98, 104101.

Girchenko, A., V. Eremeyev, and H. Altenbach, 2012, Int. J. Eng. Sci. 61, 53.

Gorkunov, M., S. A. Gredeskul, I. V. Shadrivov, and Y. S. Kivshar, 2006, Phys. Rev. E 73, 056605.

Gorkunov, M., and M. Lapine, 2004, Phys. Rev. B 70, 235109.

Gorkunov, M., M. Lapine, E. Shamonina, and K. H. Ringhofer, 2002, Eur. Phys. J. B 28, 263.

Gorkunov, M., and M. Osipov, 2008, J. Appl. Phys. 103, 036101.

Gorkunov, M., I. Shadrivov, and Y. Kivshar, 2006, Appl. Phys. Lett. 88, 071912.

Gu, J., R. Singh, Z. Tian, W. Cao, Q. Xing, M. He, J. W. Zhang, J. Han, H.-T. Chen, and W. Zhang, 2010, Appl. Phys. Lett. 97, 071102 .

Hannam, K., D. Powell, I. Shadrivov, and Y. Kivshar, 2012, Appl. Phys. Lett. 100, 081111.

Hardy, W. N., and L. A. Whitehead, 1981, Rev. Sci. Instrum. 52, 213. Harutyunyan, H., R. Beams, and L. Novotny, 2013, Nat. Phys. 9, 423.

Hegde, R., and H. Winful, 2005, Microwave Opt. Technol. Lett. 46, 528.

Herbold, E., and V. Nesterenko, 2007, Appl. Phys. Lett. 90, 261902. Herbold, E., and V. Nesterenko, 2013, Phys. Rev. Lett. 110, 144101. 
Hesmer, F., E. Tatartschuk, O. Zhuromskyy, A. A. Radkovskaya, M. Shamonin, T. Hao, C. J. Stevens, G. Faulkner, D. J. Edwards, and E. Shamonina, 2007, Phys. Status Solidi B 244, 1170.

Hodgkinson, I., Q. H. Wu, B. Knight, A. Lakhtakia, and K. Robbie, 2000, Appl. Opt. 39, 642.

Hu, Y., S. Wen, Y. Wang, and D. Fan, 2008, Opt. Commun. 281, 2663.

Hu, Y., and H. Zhuo, 2009, J. Opt. Soc. Am. B 26, B68.

Huang, D., E. Poutrina, and D. Smith, 2010, Appl. Phys. Lett. 96, 104104.

Huang, D., E. Poutrina, H. Zheng, and D. Smith, 2011, J. Opt. Soc. Am. B 28, 2925.

Huang, D., A. Rose, E. Poutrina, S. Larouche, and D. Smith, 2011, Appl. Phys. Lett. 98, 204102.

Husakou, A., and J. Herrmann, 2006, Opt. Express 14, 11194.

Husu, H., B. Canfield, J. Laukkanen, B. Bai, M. Kuittinen, J. Turunen, and M. Kauranen, 2008, Metamaterials 2, 155.

Husu, H., R. Siikanen, J. Mäkitalo, J. Lehtolahti, J. Laukkanen, M. Kuittinen, and M. Kauranen, 2012, Nano Lett. 12, 673.

Huttunen, M. J., G. Bautista, M. Decker, S. Linden, M. Wegener, and M. Kauranen, 2011, Opt. Mater. Express 1, 46.

Ilic, I., P. Belicev, V. Milanovic, J. Radovanovic, and L. Hadievski, 2011, Phys. Lett. A 375, 1357.

Iorsh, I., I. Shadrivov, P. Belov, and Y. Kivshar, 2012, Physica Status Solidi (RRL) 6, 43.

Ironside, D., and J.-T. Shen, 2013, Appl. Phys. Lett. 102, 021907.

Jin, B., et al., 2010, Opt. Express 18, 17504.

Joseph, A., and K. Porsezian, 2010, Phys. Rev. A 81, 023805.

Joseph, A., K. Porsezian, and P. P. Tchofo Dinda, 2010, J. Mod. Opt. 57, 436.

Jung, P., S. Butz, M. Marthaler, M. V. Fistul, J. Leppäkangas, V. P. Koshelets, and A. V. Ustinov, 2014, arXiv:1312.2937.

Kalinin, V. A., and V. V. Shtykov, 1990, Radiotekh. Elektron., 11, 2275 [Sov. J. Commun. Technol. Electron. 36, 96 (1991)].

Kanazawa, T., Y. Tamayama, T. Nakanishi, and M. Kitano, 2011, Appl. Phys. Lett. 99, 024101.

Kang, B., J. Woo, E. Choi, H.-H. Lee, E. Kim, J. Kim, T.-J. Hwang, Y.-S. Park, D. Kim, and J. Wu, 2010, Opt. Express 18, 16492.

Kapitanova, P., S. Maslovski, I. Shadrivov, P. Voroshilov, D. Filonov, P. Belov, and Y. Kivshar, 2011, Appl. Phys. Lett. 99, 251914.

Kapitanova, P. V., A. P. Slobozhnanyuk, I. V. Shadrivov, P. A. Belov, and Y. S. Kivshar, 2012, Appl. Phys. Lett. 101, 231904.

Katko, A., S. Gu, J. Barrett, B.-I. Popa, G. Shvets, and S. Cummer, 2010, Phys. Rev. Lett. 105, 123905.

Katko, A., A. Hawkes, J. Barrett, and S. Cummer, 2011, IEEE Antennas Wireless Propag. Lett. 10, 1571.

Katko, A., G. Shvets, and S. Cummer, 2012, J. Opt. 14, 114003.

Kauranen, M., and A. V. Zayats, 2012, Nat. Photonics 6, 737.

Kazantseva, E., A. Maimistov, and S. Ozhenko, 2009, Phys. Rev. A 80, 043833.

Khoo, I., A. Diaz, J. Liou, M. Stinger, J. Huang, and Y. Ma, 2010, IEEE J. Sel. Top. Quantum Electron. 16, 410.

Kildishev, A., 2010, Metamaterials 4, 77.

Kildishev, A., and N. Litchinitser, 2010, Opt. Commun. 283, 1628.

Kildishev, A., Y. Sivan, N. Litchinitser, and V. Shalaev, 2009, Opt. Lett. 34, 3364.

Kim, E., F. Wang, W. Wu, Z. Yu, and Y. Shen, 2008, Phys. Rev. B 78, 113102.

Kim, K., D. Phung, F. Rotermund, and H. Lim, 2008, Opt. Express 16, 1150.

Kinsler, P., 2010, Phys. Rev. A 81, 013819.
Kivshar, Y. S., and G. P. Agrawal, 2003, Optical Solitons: From Fibers to Photonic Crystals (Academic Press, San Diego).

Klein, M., C. Enkrich, M. Wegener, and S. Linden, 2006, Science 313, 502.

Klein, M., M. Wegener, N. Feth, and S. Linden, 2007, Opt. Express 15, 5238 .

Kockaert, P., P. Tassin, G. van der Sande, I. Veretennicoff, and M. Tlidi, 2006, Phys. Rev. A 74, 033822.

Kockaert, P., P. Tassin, I. Veretennicoff, G. van der Sande, and M. Tlidi, 2009, J. Opt. Soc. Am. B 26, B148.

Korotkevich, A., K. Rasmussen, G. Kovačič, V. Roytburd, A. Maimistov, and I. Gabitov, 2013, J. Opt. Soc. Am. B 30, 1077.

Kourakis, I., N. Lazarides, and G. Tsironis, 2007, Phys. Rev. E 75, 067601.

Kourakis, I., and P. Shukla, 2005, Phys. Rev. E 72, 016626.

Kozyrev, A., H. Kim, A. Karbassi, and D. van der Weide, 2005, Appl. Phys. Lett. 87, 121109.

Kozyrev, A., H. Kim, and D. van der Weide, 2006, Appl. Phys. Lett. 88, 264101.

Kozyrev, A., and D. van der Weide, 2005, in Proceedings of the IEEE Antennas and Propagation Society International Symposium (IEEE, New York), Vol. 1A, pp. 672-675.

Kozyrev, A., and D. van der Weide, 2006, Left-handed nonlinear transmission line media, U.S. Patent No. 7135917.

Kozyrev, A., and D. van der Weide, 2007, Appl. Phys. Lett. 91, 254111.

Kozyrev, A., and D. van der Weide, 2008, J. Phys. D 41, 173001.

Kozyrev, A., and D. van der Weide, 2010, Appl. Phys. Lett. 96, 104106.

Kozyrev, A. B., I. V. Shadrivov, and Y. S. Kivshar, 2014, Appl. Phys. Lett. 104, 084105.

Krutyanskiy, V., I. Kolmychek, E. Ganshina, T. Murzina, P. Evans, R. Pollard, A. Stashkevich, G. Wurtz, and A. Zayats, 2013, Phys. Rev. B 87, 035116.

Kudryashov, N., A. Maimistov, and D. Sinelshchikov, 2012, Phys. Lett. A 376, 3658.

Kudyshev, Z., I. Gabitov, and A. Maimistov, 2013, Phys. Rev. A 87, 063840.

Kujala, S., B. Canfield, M. Kauranen, Y. Svirko, and J. Turunen, 2007, Phys. Rev. Lett. 98, 167403.

Kurter, C., P. Tassin, A. Zhuravel, L. Zhang, T. Koschny, A. Ustinov, C. Soukoulis, and S. Anlage, 2012, Appl. Phys. Lett. 100, 121906.

Kuwata-Gonokami, M., N. Saito, Y. Ino, M. Kauranen, K. Jefimovs, T. Vallius, J. Turunen, and Y. Svirko, 2005, Phys. Rev. Lett. 95, 227401

Kwon, D.-H., X. Wang, Z. Bayraktar, B. Weiner, and D. H. Werner, 2008, Opt. Lett. 33, 545.

Lakes, R., 1993, Nature (London) 361, 511.

Lapine, M., and M. Gorkunov, 2004, Phys. Rev. E 70, 066601.

Lapine, M., M. Gorkunov, and K. Ringhofer, 2003, Phys. Rev. E 67, 065601.

Lapine, M., L. Jelinek, M. Freire, and R. Marqués, 2010, Phys. Rev. B 82, 165124.

Lapine, M., L. Jelinek, and R. Marqués, 2012, Opt. Express 20, 18 297.

Lapine, M., I. Shadrivov, and Y. Kivshar, 2012, Sci. Rep. 2, 412.

Lapine, M., I. Shadrivov, D. Powell, and Y. Kivshar, 2012, Nat. Mater. 11, 30.

Lapine, M., I. V. Shadrivov, D. A. Powell, and Y. S. Kivshar, 2011, Sci. Rep. 1, 138.

Lapine, M., and S. Tretyakov, 2007, IET Microwaves, Antennas and Propagation 1, 3 [http://ieeexplore.ieee.org/xpl/articleDetails.jsp? arnumber=4126160]. 
Larouche, S., A. Rose, E. Poutrina, D. Huang, and D. Smith, 2010, Appl. Phys. Lett. 97, 011109.

Larouche, S., and D. Smith, 2010, Opt. Commun. 283, 1621.

Lazarides, N., M. Eleftheriou, and G. Tsironis, 2006, Phys. Rev. Lett. 97, 157406.

Lazarides, N., M. Molina, G. Tsironis, and Y. Kivshar, 2010, Phys. Lett. A 374, 2095.

Lazarides, N., and G. Tsironis, 2005, Phys. Rev. E 71, 036614.

Lazarides, N., and G. Tsironis, 2007, Appl. Phys. Lett. 90, 163501.

Lazarides, N., G. Tsironis, and Y. Kivshar, 2008, Phys. Rev. E 77, 065601.

Leonhardt, U., and D. Smith, 2008, New J. Phys. 10, 115019.

Li, P., R. Yang, and Z. Xu, 2010, Phys. Rev. E 82, 046603.

Lim, S., C. Caloz, and T. Itoh, 2004, IEEE Trans. Microwave Theory Tech. 52, 2678.

Litchinitser, N., I. Gabitov, and A. Maimistov, 2007, Phys. Rev. Lett. 99, 113902.

Litchinitser, N., and V. Shalaev, 2008, Laser Phys. Lett. 5, 411.

Litchinitser, N. M., I. R. Gabitov, A. I. Maimistov, and V. M. Shalaev, 2007, Opt. Lett. 32, 151.

Liu, M., D. A. Powell, I. V. Shadrivov, M. Lapine, and Y. S. Kivshar, 2013, New J. Phys. 15, 073036.

Liu, M., Y. Sun, D. A. Powell, I. V. Shadrivov, M. Lapine, R. C. McPhedran, and Y. S. Kivshar, 2013, Phys. Rev. B 87, 235126.

Liu, M., et al., 2012, Nature (London) 487, 345.

Liu, Y., G. Bartal, D. Genov, and X. Zhang, 2007, Phys. Rev. Lett. 99, 153901.

Lopez, M., M. Freire, J. Algarin, V. Behr, P. Jakob, and R. Marques, 2011, Appl. Phys. Lett. 98, 133508.

Mackay, T., 2005, Electromagnetics 25, 461.

Maimistov, A., and I. Gabitov, 2007, Eur. Phys. J. Spec. Top. 147, 265.

Maimistov, A., and I. Gabitov, 2010, Opt. Commun. 283, 1633.

Maimistov, A., I. Gabitov, and A. Korotkevich, 2007, Quantum Electron. 37, 549.

Maksymov, I., A. Miroshnichenko, and Y. Kivshar, 2013, Opt. Lett. 38, 79.

Maluckov, A., L. Hadievski, N. Lazarides, and G. Tsironis, 2008, Phys. Rev. E 77, 046607.

Malyuskin, O., V. Fusco, and A. Schuchinsky, 2006a, IEEE Trans. Antennas Propag. 54, 192.

Malyuskin, O., V. Fusco, and A. Schuchinsky, 2006b, IEEE Trans. Antennas Propag. 54, 1399.

Mandelstam, L. I., 1945, Zh. Eksp. Teor. Fiz. 15, 475.

Manktelow, K., M. Leamy, and M. Ruzzene, 2011, Nonlinear Dynamics 63, 193.

Manktelow, K., R. Narisetti, M. Leamy, and M. Ruzzene, 2013, Mechanical Systems and Signal Processing 39, 32.

Marklund, M., P. Shukla, and L. Stenflo, 2006, Phys. Rev. E 73, 037601.

Marqués, R., F. Martín, and M. Sorolla, 2008, Metamaterials with negative parameters (Wiley, $\mathrm{NJ}$ ).

Martin, D., and M. Hoyuelos, 2009, Phys. Rev. E 80, 056601.

Martin, D., and M. Hoyuelos, 2010, Phys. Rev. A 82, 033841.

Mattheakis, M., G. Tsironis, and V. Kovanis, 2012, J. Opt. 14, 114006.

Mattiucci, N., G. D’Aguanno, N. Akozbek, M. Scalora, and M. Bloemer, 2010, Opt. Commun. 283, 1613.

Mattiucci, N., G. D’Aguanno, M. Bloemer, and M. Scalora, 2005, Phys. Rev. E 72, 066612.

McEnery, K., M. Tame, S. Maier, and M. Kim, 2014, Phys. Rev. A 89, 013822.
McPhedran, R. C., I. V. Shadrivov, B. T. Kuhlmey, and Y. S. Kivshar, 2011, NPG Asia Mater. 3, 100.

Milford, G., and M. Gibbons, 2011, in Proceedings of the AsiaPacific Microwave Conference (APMC-2011), pp. 538-541.

Minovich, A., D. Neshev, D. Powell, I. Shadrivov, and Y. Kivshar, 2010, Appl. Phys. Lett. 96, 193103.

Minovich, A., et al., 2012, Appl. Phys. Lett. 100, 121113.

Nakanishi, T., Y. Tamayama, and M. Kitano, 2012, Appl. Phys. Lett. 100, 044103.

Narahara, K., 2007, Jpn. J. Appl. Phys. 46, 5353.

Narahara, K., T. Nakamichi, T. Otsuji, and E. Sano, 2007, Jpn. J. Appl. Phys. 46, 3123.

Nicolson, A. M., and G. F. Ross, 1970, IEEE Trans. Instrum. Meas. 19, 377.

Niesler, F., N. Feth, S. Linden, J. Niegemann, J. Gieseler, K. Busch, and M. Wegener, 2009, Opt. Lett. 34, 1997.

Nikolaenko, A., F. de Angelis, S. Boden, N. Papasimakis, P. Ashburn, E. di Fabrizio, and N. Zheludev, 2010, Phys. Rev. Lett. 104, 153902.

Nikolaenko, A., N. Papasimakis, E. Atmatzakis, Z. Luo, Z. Shen, F. De Angelis, S. Boden, E. Di Fabrizio, and N. Zheludev, 2012, Appl. Phys. Lett. 100, 181109.

Noginov, M., M. Lapine, V. Podolskiy, and Y. Kivshar, 2013, Opt. Express 21, 14895.

Noskov, R., and A. Zharov, 2006, Opto-Electron. Rev. 14, 217.

O'Brien, S., D. McPeake, S. Ramakrishna, and J. Pendry, 2004, Phys. Rev. B 69, 241101.

Ostroukhova, E. I., and A. I. Maimistov, 2012, Opt. Spectrosc. 112, 255.

Padilla, W. J., A. J. Taylor, C. Highstrete, M. Lee, and R. D. Averitt, 2006, Phys. Rev. Lett. 96, 107401.

Pafomov, V. E., 1959, JETP 36, 1853 [Sov. Phys. JETP 9, 1321 (1959)].

Palomba, S., S. Zhang, Y. Park, G. Bartal, X. Yin, and X. Zhang, 2012, Nat. Mater. 11, 34.

Paul, T., C. Rockstuhl, and F. Lederer, 2010, J. Opt. Soc. Am. B 27, 1118.

Paul, T., C. Rockstuhl, and F. Lederer, 2011, J. Mod. Opt. 58, 438.

Pendry, J. B., 2000, Phys. Rev. Lett. 85, 3966.

Pendry, J. B., 2011, New Sci. 209, ii.

Pendry, J. B., A. J. Holden, D. J. Robbins, and W. J. Stewart, 1999, IEEE Trans. Microwave Theory Tech. 47, 2075.

Pimenov, A., A. Loidl, P. Przyslupski, and B. Dabrowski, 2005, Phys. Rev. Lett. 95, 247009.

Popov, A., 2010, Eur. Phys. J. D 58, 263.

Popov, A., and S. Myslivets, 2011, Appl. Phys. A 103, 725.

Popov, A., S. Myslivets, T. George, and V. Shalaev, 2007, Opt. Lett. 32, 3044.

Popov, A., M. Shalaev, S. Myslivets, V. Slabko, and I. Nefedov, 2012, Appl. Phys. A 109, 835.

Popov, A., and V. Shalaev, 2006a, Opt. Lett. 31, 2169.

Popov, A., and V. Shalaev, 2006b, Appl. Phys. B 84, 131.

Popov, A. K., S. A. Myslivets, and V. M. Shalaev, 2009, Opt. Lett. 34, 1165.

Popov, A. K., V. V. Slabko, and V. M. Shalaev, 2006, Laser Phys. Lett. 3, 293.

Poutrina, E., D. Huang, and D. R. Smith, 2010, New J. Phys. 12, 093010.

Poutrina, E., D. Huang, Y. Urzhumov, and D. Smith, 2011, Opt. Express 19, 8312.

Powell, D., K. Hannam, I. Shadrivov, and Y. Kivshar, 2011, Phys. Rev. B 83, 235420. 
Powell, D., M. Lapine, M. Gorkunov, I. Shadrivov, and Y. Kivshar, 2010, Phys. Rev. B 82, 155128.

Powell, D., I. Shadrivov, and Y. Kivshar, 2008, Appl. Phys. Lett. 92, 264104.

Powell, D., I. Shadrivov, and Y. Kivshar, 2009a, Appl. Phys. Lett. 94, 084105.

Powell, D., I. Shadrivov, and Y. Kivshar, 2009b, Appl. Phys. Lett. 95, 084102.

Powell, D., I. Shadrivov, Y. Kivshar, and M. Gorkunov, 2007, Appl. Phys. Lett. 91, 144107.

Quach, J., C.-H. Su, A. Martin, A. Greentree, and L. Hollenberg, 2011, Opt. Express 19, 11018.

Rahm, M., J.-S. Li, and W. Padilla, 2013, Journal of Infrared, Millimeter, and Terahertz Waves 34, 1.

Rakhmanov, A., A. Zagoskin, S. Savel'ev, and F. Noti, 2008, Phys. Rev. B 77, 144507.

Rapoport, Y., V. Grimalsky, I. Iorsh, N. Kalinich, S. Koshevaya, C. Castrejon-Martinez, and Y. Kivshar, 2013, JETP Lett. 98, 503.

Reinhold, J., et al., 2012, Phys. Rev. B 86, 115401.

Ren, M., E. Plum, J. Xu, and N. Zheludev, 2012, Nat. Commun. 3, 833.

Reynet, O., and O. Acher, 2004, Appl. Phys. Lett. 84, 1198.

Ricci, M., N. Orloff, and S. M. Anlage, 2005, Appl. Phys. Lett. 87, 034102.

Ricci, M. C., and S. M. Anlage, 2006, Appl. Phys. Lett. 88, 264102.

Ricci, M. C., H. Xu, R. Prozorov, A. P. Zhuravel, A. V. Ustinov, and S. M. Anlage, 2007, IEEE Trans. Appl. Supercond. 17, 918.

Rico-García, J., J. López-Alonso, and A. Aradian, 2012, J. Opt. Soc. Am. B 29, 53.

Rogacheva, A., V. Fedotov, A. Schwanecke, and N. Zheludev, 2006, Phys. Rev. Lett. 97, 177401.

Roppo, V., M. Centini, D. de Ceglia, M. Vicenti, J. Haus, N. Akozbek, M. Bloemer, and M. Scalora, 2008, Metamaterials 2, 135.

Roppo, V., C. Ciraci, C. Cojocaru, and M. Scalora, 2010, J. Opt. Soc. Am. B 27, 1671.

Roppo, V., et al., 2007, Phys. Rev. A 76, 033829.

Rosanov, N., N. Vysotina, A. Shatsev, I. Shadrivov, and Y. Kivshar, 2011a, JETP Lett. 93, 743.

Rosanov, N., N. Vysotina, A. Shatsev, I. Shadrivov, D. Powell, and Y. Kivshar, 2011b, Opt. Express 19, 26500.

Rosanov, N. N., N. V. Vysotina, A. N. Shatsev, A. S. Desyatnikov, and Y. S. Kivshar, 2012, Phys. Rev. Lett. 108, 133902.

Rose, A., D. Huang, and D. Smith, 2011, Phys. Rev. Lett. 107, 063902.

Rose, A., D. Huang, and D. Smith, 2012, Appl. Phys. Lett. 101, 051103.

Rose, A., S. Larouche, D. Huang, E. Poutrina, and D. Smith, 2010, Phys. Rev. E 82, 036608.

Rose, A., S. Larouche, E. Poutrina, and D. Smith, 2012, Phys. Rev. A 86, 033816.

Rose, A., S. Larouche, and D. Smith, 2011, Phys. Rev. A 84, 053805 .

Rose, A., D. A. Powell, I. V. Shadrivov, D. R. Smith, and Y. S. Kivshar, 2013, Phys. Rev. B 88, 195148.

Rose, A., and D. R. Smith, 2011, Phys. Rev. A 84, 013823.

Ruppin, R., 2000, Phys. Lett. A 277, 61.

Ryzhov, M., and A. Maimistov, 2012, Quantum Electron. 42, 1034.

Sarma, A., 2011, Eur. Phys. J. D 62, 421.

Sarma, A., and M. Saha, 2011, J. Opt. Soc. Am. B 28, 944.

Savinov, V., V. A. Fedotov, S. M. Anlage, P. A. J. de Groot, and N. I. Zheludev, 2012, Phys. Rev. Lett. 109, 243904.
Scalora, M., G. D’Aguanno, M. Bloemer, M. Centini, D. de Ceglia, N. Mattiucci, and Y. Kivshar, 2006, Opt. Express 14, 4746.

Scalora, M., D. de Ceglia, G. D’Aguanno, N. Mattiucci, N. Akozbek, M. Centini, and M. Bloemer, 2007, Phys. Rev. E 75, 066606.

Scalora, M., M. Syrchin, N. Akozbek, E. Poliakov, G. D’Aguanno, N. Mattiucci, M. Bloemer, and A. Zheltikov, 2005, Phys. Rev. Lett. 95, 013902.

Scalora, M., M. Vincenti, D. de Ceglia, V. Roppo, M. Centini, N. Akozbek, and M. Bloemer, 2010, Phys. Rev. A 82, 043828.

Shadrivov, I., 2004, Photonics and Nanostructures: Fundamentals and Applications 2, 175.

Shadrivov, I., 2012, Appl. Phys. Lett. 101, 041911.

Shadrivov, I., V. Fedotov, D. Powell, Y. Kivshar, and N. Zheludev, 2011, New J. Phys. 13, 033025.

Shadrivov, I., and Y. Kivshar, 2005, J. Opt. A 7, S68.

Shadrivov, I., A. Kozyrev, D. van der Weide, and Y. Kivshar, 2008a, Opt. Express 16, 20266.

Shadrivov, I., A. Kozyrev, D. van der Weide, and Y. Kivshar, 2008b, Appl. Phys. Lett. 93, 161903.

Shadrivov, I., S. Morrison, and Y. Kivshar, 2006, Opt. Express 14, 9344.

Shadrivov, I., A. Sukhorukov, Y. Kivshar, A. Zharov, A. Boardman, and P. Egan, 2004, Phys. Rev. E 69, 016617.

Shadrivov, I., A. Zharov, and Y. Kivshar, 2006, J. Opt. Soc. Am. B 23, 529.

Shadrivov, I., A. Zharov, N. Zharova, and Y. Kivshar, 2006, Photonics and Nanostructures Fundamentals and Applications 4, 69.

Shadrivov, I. V., P. V. Kapitanova, S. I. Maslovski, and Y. S. Kivshar, 2012, Phys. Rev. Lett. 109, 083902.

Shalaev, V. M., 2007, Nat. Photonics 1, 41.

Shamonina, E., V. A. Kalinin, K. H. Ringhofer, and L. Solymar, 2002, J. Appl. Phys. 92, 6252.

Shamonina, E., and L. Solymar, 2007, Metamaterials 1, 12.

Shramkova, O., and A. Schuchinsky, 2012, Int. J. RF and Microwave CAE 22, 469.

Sievenpiper, D., 2011, IEEE Antennas Wireless Propag. Lett. 10, 1516.

Sihvola, A., 2007, Metamaterials 1, 2.

Silin, R. A., 2012, Metamaterials 6, 1.

Silin, R. A., and V.P. Sazonow, 1971, Slow-wave structure (Staschera, Boston-SPA Eng., Boston).

Silveirinha, M., J. Baena, L. Jelinek, and R. Marques, 2009, Metamaterials 3, 115.

Simovski, C. R., 2009, Opt. Spectrosc. 107, 726.

Skarka, V., N. B. Aleksić, and V. I. Berezhiani, 2010, Phys. Rev. A 81, 045803.

Slobozhanyuk, A. P., P. V. Kapitanova, D. S. Filonov, D. A. Powell, I. V. Shadrivov, M. Lapine, P. A. Belov, R. C. McPhedran,

and Y. S. Kivshar, 2014, Appl. Phys. Lett. 104, 014104.

Slobozhanyuk, A. P., M. Lapine, D. Powell, I. V. Shadrivov, Y. S. Kivshar, R. C. McPhedran, and P. A. Belov, 2013, Adv. Mater. 25, 3409.

Smith, D. R., 2004, Phys. World 17, 23.

Smith, D. R., 2010, Phys. Rev. E 81, 036605.

Smith, D. R., W. J. Padilla, D. C. Vier, S. C. Nemat-Nasser, and S. Schultz, 2000, Phys. Rev. Lett. 84, 4184.

Smith, D. R., S. Schultz, P. Markoš, and C. M. Soukoulis, 2002, Phys. Rev. B 65, 195104.

Smith, D. R., D. C. Vier, N. Kroll, and S. Schultz, 2000, Appl. Phys. Lett. 77, 2246. 
Soljačić, M., M. Ibanescu, S. G. Johnson, Y. Fink, and J. D. Joannopoulos, 2002, Phys. Rev. E 66, 055601.

Solymar, L., and E. Shamonina, 2009, Waves in Metamaterials (Oxford University Press, Oxford, United Kingdom).

Somerville, W., D. Powell, and I. Shadrivov, 2011, Appl. Phys. Lett. 98, 161111.

Suchowski, H., K. O’Brien, Z. J. Wong, A. Salandrino, X. Yin, and X. Zhang, 2013, Science 342, 1223.

Sydoruk, O., V. Kalinin, and E. Shamonina, 2007, Phys. Status Solidi (b) 244, 1176.

Sydoruk, O., A. Radkovskaya, O. Zhuromskyy, E. Shamonina, M. Shamonin, C. J. Stevens, G. Faulkner, D. J. Edwards, and L. Solymar, 2006, Phys. Rev. B 73, 224406.

Sydoruk, O., E. Shamonina, and L. Solymar, 2007, J. Phys. D 40, 6879.

Sydoruk, O., O. Zhuromskyy, E. Shamonina, and L. Solymar, 2005, Appl. Phys. Lett. 87, 072501.

Syms, R., L. Solymar, and I. Young, 2008, Metamaterials 2, 122.

Syms, R. R. A., E. Shamonina, V. Kalinin, and L. Solymar, 2005, J. Appl. Phys. 97, 064909.

Syms, R. R. A., and L. Solymar, 2011, J. Appl. Phys. 109, 124909.

Syms, R. R. A., O. Sydoruk, and L. Solymar, 2011, Phys. Rev. B 84, 235150.

Tankeyev, A., V. Smagin, M. Borich, and A. Zhuravlev, 2009, Phys. Met. Metallogr. 107, 229.

Tassin, P., G. van der Sande, N. Veretenov, P. Kockaert, I. Yeretennicoff, and M. Tlidi, 2006, Opt. Express 14, 9338.

Torner, L., and Y. Kartashov, 2009, Opt. Lett. 34, 1129.

Toroghi, S., and P. Kik, 2012, Phys. Rev. B 85, 045432.

Trepanier, M., D. Zhang, O. Mukhanov, and S. Anlage, 2013, Phys. Rev. X 3, 041029.

Tsitsas, N., T. Horikis, Y. Shen, P. Kevrekidis, N. Whitaker, and D. Frantzeskakis, 2010, Phys. Lett. A 374, 1384.

Tsurumi, T., 2008, J. Phys. Soc. Jpn. 77, 074006.

Tuovinen, H., M. Kauranen, K. Jefimovs, P. Vahimaa, T. Vallius, J. Turunen, N. V. Tkachenko, and H. Lemmetyinen, 2002, J. Nonlinear Opt. Phys. Mater. 11, 421.

Tuz, V., S. Prosvirnin, and L. Kochetova, 2010, Phys. Rev. B 82, 233402.

Valev, V., J. Baumberg, C. Sibilia, and T. Verbiest, 2013, Adv. Mater. 25, 2517.

Valev, V., et al., 2011, ACS Nano 5, 91.

Valev, V. K., et al., 2013, Opt. Lett. 38, 2256.

van der Ziel, J. P., and M. Ilegems, 1976, Appl. Phys. Lett. 28, 437.

Veronis, G., and S. Fan, 2009, Proc. SPIE Int. Soc. Opt. Eng. 7218, $72180 \mathrm{Y}$

Veselago, V. G., 1968, Soviet Physics Uspekhi 10, 509 Usp. Fiz. Nauk 92, 517 (1967)].

Vincenti, M., S. Campione, D. de Ceglia, F. Capolino, and M. Scalora, 2012, New J. Phys. 14, 103016.

Wang, B., J. Zhou, T. Koschny, and C. Soukoulis, 2008, Opt. Express 16, 16058.

Wang, F., F. Rodriguez, W. Albers, R. Ahorinta, J. Sipe, and M. Kauranen, 2009, Phys. Rev. B 80, 233402.

Wang, Z., Y. Feng, B. Zhu, J. Zhao, and T. Jiang, 2010, J. Appl. Phys. 107, 094907.

Wang, Z., Y. Luo, T. Jiang, Z. Wang, J. Huangfu, and L. Ran, 2011, Phys. Rev. Lett. 106, 047402.

Wei, R.-R., X. Chen, J.-W. Tao, and C.-F. Li, 2008, Phys. Lett. A 372, 6797.
Weir, W. B., 1974, Proc. IEEE 62, 33.

Wen, S., Y. Wang, W. Su, Y. Xiang, X. Fu, and D. Fan, 2006, Phys. Rev. E 73, 036617.

Wen, S., Y. Xiang, X. Dai, Z. Tang, W. Su, and D. Fan, 2007, Phys. Rev. A 75, 033815.

Werner, D. H., D.-H. Kwon, I. C. Khoo, A. V. Kildishev, and V. M. Shalaev, 2007, Opt. Express 15, 3342.

Winful, H. G., J. H. Marburger, and E. Garmire, 1979, Appl. Phys. Lett. 35, 379.

Wokaun, A., J. G. Bergman, J. P. Heritage, A. M. Glass, P. F. Liao, and D. H. Olson, 1981, Phys. Rev. B 24, 849.

Wurtz, G., R. Pollard, W. Hendren, G. Wiederrecht, D. Gosztola, V. Podolskiy, and A. Zayats, 2011, Nat. Nanotechnol. 6, 107.

Xiang, Y., X. Dai, S. Wen, and D. Fan, 2011, J. Opt. Soc. Am. B 28, 908.

Xiang, Y., S. Wen, X. Dai, and D. Fan, 2010, Phys. Rev. E 82, 056605.

Xiang, Y., S. Wen, X. Dai, Z. Tang, W. Su, and D. Fan, 2007, J. Opt. Soc. Am. B 24, 3058.

Xiao, S., U. Chettiar, A. Kildishev, V. Drachev, I. Khoo, and V. Shalaev, 2009, Appl. Phys. Lett. 95, 033115.

Xu, G., T. Pan, T. Zang, and J. Sun, 2009, J. Phys. D 42, 045303.

Yang, R., and I. Shadrivov, 2010, Appl. Phys. Lett. 97, 231114.

Yang, R., and Y. Zhang, 2011, J. Opt. Soc. Am. B 28, 123.

Yannopapas, V., 2010, Opt. Commun. 283, 1647.

Yomba, E., 2008, Phys. Lett. A 372, 1612.

Zagoskin, A., 2011, Quantum Engineering (Cambridge University Press, Cambridge, England).

Zdanowicz, M., S. Kujala, H. Husu, and M. Kauranen, 2011, New J. Phys. 13, 023025.

Zeng, Y., D. Dalvit, J. O’Hara, and S. Trugman, 2012, Phys. Rev. B 85, 125107.

Zeng, Y., W. Hoyer, J. Liu, S. Koch, and J. Moloney, 2009, Phys. Rev. B 79, 235109.

Zhang, C., B. Jin, J. Han, I. Kawayama, H. Murakami, J. Wu, L. Kang, J. Chen, P. Wu, and M. Tonouchi, 2013, Appl. Phys. Lett. 102, 081121 .

Zhang, F., L. Kang, Q. Zhao, J. Zhou, X. Zhao, and D. Lippens, 2009, Opt. Express 17, 4360.

Zhang, F., W. Zhang, Q. Zhao, J. Sun, K. Qiu, J. Zhou, and D. Lippens, 2011, Opt. Express 19, 1563.

Zhang, F., Q. Zhao, L. Kang, D. Gaillot, Z. Zhao, J. Zhou, and D. Lippens, 2008, Appl. Phys. Lett. 92, 193104.

Zhang, F., Q. Zhao, W. Zhang, J. Sun, J. Zhou, and D. Lippens, 2010, Appl. Phys. Lett. 97, 134103.

Zhang, J., K. F. MacDonald, and N. I. Zheludev, 2013, Light Sci. Appl. 2, e96.

Zhang, J., S. Wen, Y. Xiang, and H. Luo, 2010, J. Mod. Opt. 57, 876.

Zhang, J., S. Wen, Y. Xiang, Y. Wang, and H. Luo, 2010, Phys. Rev. A 81, 023829.

Zhang, L.-J., L. Chen, and C.-H. Liang, 2008, J. Electromagn. Waves Appl. 22, 1031.

Zhang, W., Y. Chen, P. Hou, J. Shi, and Q. Wang, 2010, Phys. Rev. E 82, 066601.

Zhao, Q., L. Kang, B. Du, B. Li, J. Zhou, H. Tang, X. Liang, and B. Zhang, 2007, Appl. Phys. Lett. 90, 011112.

Zharov, A., I. Shadrivov, and Y. Kivshar, 2003, Phys. Rev. Lett. 91, 037401 . 
Zharov, A., and N. Zharova, 2010, JETP Lett. 92, 210.

Zharov, A., N. Zharova, I. Shadrivov, and Y. Kivshar, 2005, Appl.

Phys. Lett. 87, 091104.

Zharova, N., I. Shadrivov, A. Zharov, and Y. Kivshar, 2005, Opt. Express 13, 1291.

Zheludev, N., and V. Emel'yanov, 2004, J. Opt. A 6, 26.

Zheludev, N., and Y. Kivshar, 2012, Nat. Mater. 11, 917.
Zheludev, N. I., 2011, Opt. Photonics News 22, 30.

Zueco, D., C. Fernández-Juez, J. Yago, U. Naether, B. Peropadre, J. García-Ripoll, and J. Mazo, 2013, Supercond. Sci. Technol. 26, 074006.

Zyablovsky, A., A. Dorofeenko, A. Vinogradov, and A. Pukhov, 2011, Photonics and Nanostructures Fundamentals and Applications 9, 398. 